\title{
Release sites are positioned to activate NMDA receptors
}

Shuo Li ${ }^{1,2}$, Sumana Raychaudhuri ${ }^{1}$, Stephen Alexander Lee ${ }^{3}$, Jing Wang ${ }^{4}$, Grant Kusick ${ }^{1,5}$, ${\text { Christine } \text { Prater }^{3 \#^{*}}, \text { Sarah Syed }}^{1^{*}}$, Hanieh Falahati ${ }^{3 \wedge *}$, Raul Ramos $^{3 \dagger^{* *}}$, Tomas M. Bartol ${ }^{6}$, Eric Hosy $^{7,8}$, Shigeki Watanabe ${ }^{1,9}$

${ }^{1}$ Department of Cell Biology, Johns Hopkins University, School of Medicine, Baltimore, MD 21205, USA

${ }^{2}$ Department of Biological and Molecular Biology, Johns Hopkins Bloomberg School of Public Health, Baltimore MD 21205,

${ }^{3}$ Neurobiology Course, The Marine Biological Laboratory, Woods Hole, MA 02543, USA.

${ }^{4}$ ThermoFisher Scientific, Hillsboro, OR 97125

${ }^{5}$ Biochemistry, Cellular and Molecular Biology Graduate Program, Johns Hopkins University, School of Medicine, Baltimore, MD 21205, USA.

${ }^{6}$ Howard Hughes Medical Institute, Salk Institute for Biological Studies, La Jolla, CA 92037 , USA

${ }^{7}$ Interdisciplinary Institute for Neuroscience, UMR 5297, Centre National de la Recherche Scientifique, 33077 Bordeaux, France

${ }^{8}$ Interdisciplinary Institute for Neuroscience, University of Bordeaux, 33077 Bordeaux, France

${ }^{9}$ Solomon H. Snyder Department of Neuroscience, Johns Hopkins University School of

Medicine, Baltimore, MD 21205, USA

Correspondence:

Shigeki Watanabe (shigeki.watanabe@jhmi.edu)

${ }^{\circ}$ Current address: Department of Biomedical Engineering, Columbia University, New York, NY 10032, USA.

\# Current address: Department of Pharmacology and Neuroscience, School of Medicine, Texas

Tech University Health Sciences Center, Lubbock, TX, 79430 USA

^ Current address: Department of Neuroscience, Yale University School of Medicine, New Haven, CT 06510

$\dagger$ Current address: Department of Biology, Brandeis University, Waltham, MA 02454

* These authors contributed equally to this work.

Key words: synaptic vesicle exocytosis, synchronous release, asynchronous release, transsynaptic alignment, AMPA receptors, NMDA receptors, zap-and-freeze, SMASH, synaptic transmission simulations, spin-mill serial-block face SEM 


\begin{abstract}
Neurotransmitter is released synchronously and asynchronously following an action potential. The release sites of these two phases are segregated within an active zone, with asynchronous release sites enriched near the center. Here we demonstrate that synchronous and asynchronous release sites are aligned with AMPA receptor and NMDA receptor clusters, respectively. Computational simulations indicate that this spatial and temporal arrangement of release ensures maximal membrane depolarization through AMPA receptors, alleviating the pore-blocking magnesium leading to greater activation of NMDA receptors. Together, these results suggest that release sites are organized to efficiently activate NMDA receptors.
\end{abstract}




\section{Main}

Neurotransmitter is released synchronously within a millisecond of an action potential and asynchronously several milliseconds later ${ }^{1}$. Both phases of release result from exocytosis of synaptic vesicles at a specialized membrane domain: the active zone ${ }^{2}$. Within the active zone lie one or more release sites, individual units at which a single synaptic vesicle may fuse ${ }^{3}$. Our recent study suggests that release sites for synchronous and asynchronous release occupy unique domains within an active zone: synchronous release sites are uniformly distributed, while asynchronous release sites are abundant near the center of an active zone ${ }^{4}$. However, the functional importance of this spatial organization is unknown.

A large body of work suggests that location of release is important for the activation of receptors. For excitatory signaling in the central nervous system, glutamate released from presynaptic boutons activates receptors on the postsynaptic membrane. Two ionotropic receptors are critical: $\alpha$-amino-3-hydroxy-5-methyl-4-isoxazolepropionic acid (AMPA) receptors and Nmethyl-D-aspartate (NMDA) receptors ${ }^{5,6}$. Given the low sensitivity of these receptors for glutamate binding ${ }^{7,8,9,10}$, the activation of receptors requires a high concentration of glutamate in the synaptic cleft, which is only achieved at the point of release due to rapid diffusion of glutamate $^{11,12,13}$. In fact, recent studies demonstrate that release sites are aligned with clusters of AMPA receptors ${ }^{14}$, and their association through trans-synaptic adhesion proteins affects the magnitude of synaptic transmission ${ }^{15}$. Thus, where glutamate is released relative to receptors is important for their activation.

The timing of glutamate release is also a key component for activation, particularly of the NMDA receptors. NMDA receptors require binding of two glutamate molecules for the activation $^{16,17}$. Depending on the concentration of glutamate, only single binding site may be 
occupied, leading to desensitization rather than activation ${ }^{16,17}$. However, the single-bound state can last for tens of milliseconds, during which the second release can favor their activation ${ }^{16,17}$. In addition, at resting membrane potential the pore of NMDA receptors is blocked by magnesium ions, requiring the removal of the block by membrane depolarization ${ }^{18,19,20}$. Classically, paired stimuli are applied for NMDA receptor activation ${ }^{21-23}$. However, a single stimulus can potentially prime NMDA receptors for activation with synchronous release, depolarizing the postsynaptic membrane and asynchronous release, providing extra glutamate in the synaptic cleft. Thus, both the locations and timing of glutamate release determine how signals are transmitted at the excitatory synapses.

To reveal the spatial and temporal relationship between release sites and receptors, we need an approach to visualize receptors relative to fusions events observed by electron microscopy. To this end, we developed a method to label these receptors with gold particles using a high-affinity interaction between nickel and polyhistidine (His-tag, hereafter; Fig.1a) ${ }^{24}$. GluA2 (AMPA receptor subunit) tagged on its extracellular domain with His-tag or HaloTag ${ }^{25}$ was expressed in the cultured wild-type mouse hippocampal neurons using lentivirus. HaloTag::GluA2 served as a negative control to test the specificity of Ni-NTA-gold labelling. We incubated these neurons with Ni-NTA-gold (5 nM) for $30 \mathrm{~min}$ and subjected them to highpressure freezing. Frozen samples were then prepared for electron microscopy, and 40-nm thick sections collected. About 100 electron micrographs were acquired per experiment from each condition and quantified blinded to treatment (Supplementary Fig.1a for example micrographs), and each experiment was repeated three times. In the wild-type neurons expressing Histag::GluA2, approximately $70 \%$ of synaptic profiles contained gold particles in the synaptic cleft; the median number of gold particles was 2.6 per synaptic profile (Supplementary Fig. 
1c,d). In contrast, almost no gold particles were observed in controls expressing HaloTag::GluA2 ( 4\% synaptic profiles, 0.03 gold/synapse, Supplementary Fig. 1a,c-d, Supplementary Table 1 for details), suggesting that the labelling is specific. We next repeated the same experiments in GluA2 knockout neurons (Gria2 $\left.2^{-/}\right)$to test if the overexpression of the GluA2 in wild type would significantly change the number of receptors at the postsynaptic density. No differences were observed between wild-type and knock-out neurons

(Supplementary Fig. 1b-f). These results suggest that this affinity-based labelling can report the distribution of receptors at the ultrastructural level. We named this approach small-metal affinity staining of His-tag, or SMASH.

With the labelling approach validated, we next measured the locations of AMPA receptors and NMDA receptors within the post-synaptic density. We expressed either Histag::GluA2 or His-tag::NR1 in wild-type neurons. In single profiles, AMPA receptors were biased towards the edge of the postsynaptic density $(\mathrm{p}<0.001)$, whereas NMDA receptors were uniformly distributed (Fig. 1b-d; $p>0.8$ ), suggesting they may occupy different domains within the postsynaptic density (Fig. 1b-d; $\mathbf{p}<0.001$ ).

To more accurately localize receptors, we reconstructed synapses using spin-mill serial block face imaging (Fig. 1e and Supplementary Fig. 2, $\mathrm{N}=2$; see Methods for details). Unlike traditional serial block face imaging approaches, spin milling using an oxygen plasma ion beam enables imaging of large areas $(400 \mu \mathrm{m} \times 400 \mu \mathrm{m})$ with isotropic resolution of $\sim 5 \mathrm{~nm}^{26}$. At least 17 synapses were reconstructed from each sample, and experiments repeated from two independent samples. Similar to single profiles, AMPA and NMDA receptors were differentially distributed in the reconstructed postsynaptic densities (Fig. 1f, $\mathrm{p}<0.001)$. As in single profiles (Fig. 1d), AMPA receptors were biased towards the edge (Fig. 1f, p $<0.001$, Supplementary 
Fig. 2a for more example). Interestingly, NMDA receptors were enriched around the center of the postsynaptic density (Fig. 1e,f, p $<0.001$, Supplementary Fig. 2c for more example). The distributions of these receptors are consistent with previous experiments using single-molecule localization microscopy ${ }^{27}$. These data suggest that AMPA receptors and NMDA receptors are differentially localized within the postsynaptic density.

Both AMPA and NMDA receptors qualitatively seemed clustered ${ }^{27}$ in the reconstructed synapses. To test whether the particles are clustered, we performed K-means cluster analysis on the reconstructed synapses (Fig. 1g,j). The same analysis was then repeated 50 times with particle locations randomized within the postsynaptic density for each synapse. The sums of square differences were compared between the actual locations and randomized locations (see Methods for detail). The median numbers of the AMPA receptor and NMDA receptor clusters were 2 and 1 per synapse, respectively, based on the K-means analysis (Fig. 1i). The median numbers of AMPA and NMDA receptors were 16 and 10 per synapse (Fig. 1k), or 470 and 274 per $\mu \mathrm{m}^{2}$, respectively (Fig. 11). These numbers are comparable to previous estimates from the freeze-fracture immuno-gold labelling of adult rat cerebellum ${ }^{28}$ and immuno-electron microscopy of rat hippocampus ${ }^{29}$. These results suggest that AMPA receptors tend to form $\sim 2$ clusters (Fig. 1i) and surround the NMDA receptor cluster, which is located near the center of the postsynaptic density.

Recent studies suggest that release sites are trans-synaptically aligned with AMPA receptors ${ }^{14}$. Our data indicate that NMDA receptors occupy different domains within the postsynaptic density (Fig. 1d,f). Thus, it is not clear if NMDA receptors also align with release sites. Interestingly, their distribution near the center of the postsynaptic density mirrors the recently described distribution of asynchronous release sites in the active zone ${ }^{4}$. To test whether 
asynchronous release sites and NMDA receptors are aligned, we performed zap-and-freeze experiments after SMASH labelling. Neurons expressing SnapTag::GluA2 or SnapTag::NR1 were used as controls for background gold staining (Supplementary Fig. 3e,f). Specifically, we stimulated neurons expressing fusion proteins once and froze them after 5 and $11 \mathrm{~ms}$. Based on our recent study, fusion intermediates captured at these time points represent synchronous and asynchronous release, respectively ${ }^{4}$. The numbers and distributions of docked vesicles and exocytic pits were all consistent with our previous studies (Fig. 2c, Supplementary Fig. $\mathbf{3 g}, \mathbf{h})^{4,30,31}$. Of note, exocytic pits at $5 \mathrm{~ms}$ were distributed throughout the active zone with a slight bias to the center (median $=0.4$ ), while fusion intermediates at $11 \mathrm{~ms}$ were strongly biased towards the center (Fig. 2a-d, median $=0.1, \mathrm{p}<0.001$, Supplementary Fig. 3a-d for more example micrographs). Thus, the distribution of fusion events during asynchronous release is similar to that of NMDA receptors.

To test the spatial relationship between fusion events and receptors, we measured the distance between receptors and docked vesicles or exocytic pits. The median distances from docked vesicles to AMPA and NMDA receptors were $95 \mathrm{~nm}$ and $73 \mathrm{~nm}$, respectively, at rest (Fig. 2e inset), and remained largely unchanged following stimulation (Fig 2e inset: AMPA receptors, $102 \mathrm{~nm}$ at $5 \mathrm{~ms}$; and $92 \mathrm{~nm}$ at $11 \mathrm{~ms}$; Fig. 2e inset: NMDA receptors, $63 \mathrm{~nm}$ at $5 \mathrm{~ms}$, $88 \mathrm{~nm}$ at $11 \mathrm{~ms}$ ). In contrast, exocytic pits were found closer to AMPA receptors at $5 \mathrm{~ms}$ (median $=67 \mathrm{~nm})$ and more distant at $11 \mathrm{~ms}($ Fig. 2f, median $=120 \mathrm{~nm}, \mathrm{p}<0.001)$. Interestingly, exocytic pits were distant from NMDA receptors at $5 \mathrm{~ms}($ median $=139 \mathrm{~nm})$ but closer at $11 \mathrm{~ms}(\mathbf{F i g . ~ 2 f}$, median $=56 \mathrm{~nm}, \mathrm{p}<0.001)$. These results suggest that neurotransmitter is released synchronously near AMPA receptors and asynchronously around NMDA receptors. 
The spatial organization of release sites and receptors and temporal sequence of their usage could allow preferential activation of NMDA receptors. First, synchronous release activates AMPA receptors, depolarizing the postsynaptic membrane and alleviating $\mathrm{Mg}^{2+}$ block of NMDA receptors ${ }^{18,19}$. Asynchronous release then increases the glutamate concentration in the synaptic cleft to favor the activation of NMDA receptors, potentially of those in the single-bound state. To test this possibility, we need to assess how NMDA receptors respond to asynchronous release in the absence of a $\mathrm{Mg}^{2+}$ block and compare it to the response following AMPA receptormediated membrane depolarization in the presence of $\mathrm{Mg}^{2+}$ block. In addition, the locations of synchronous and asynchronous release must be swapped to assess the importance of the spatial organization. To address these points, we performed computational simulations using the MCell platform that we have recently developed ${ }^{13,15}$. This model simulates receptor activation in dendritic spines by incorporating realistic synapse morphology as well as the measured kinetics of molecules ${ }^{13,15}$. We further incorporated the observed distributions of receptors (Fig. 1): clusters containing $\sim 20$ AMPA receptors and $~ 15$ NMDA receptors were placed around the periphery and center of the postsynaptic density, respectively. The centroid to centroid distance between the clusters was set at $100 \mathrm{~nm}^{15}$. We simulated the activation of AMPA and NMDA receptors with a sequence of two release events, with one occurring near the AMPA receptor cluster and another occurring near the NMDA receptor cluster. The timing of these two release events was varied from $0 \mathrm{~ms}$ to $50 \mathrm{~ms}$ apart ( 0 ms means simultaneous release at these two locations).

Using this model, we first determined how AMPA and NMDA receptors behave in response to asynchronous release in the absence of $\mathrm{Mg}^{2+}$. Asynchronous release increased the responses of AMPA receptors by $50 \%$ and NMDA receptors by $84 \%$, when compared to the 
responses from a single release (Supplementary Fig. 4a-f). For AMPA receptors, the desensitization of the receptors ${ }^{32,33}$ hampered their response to asynchronous release during the first 15 ms (Supplementary Fig. 4a-c). In contrast, the responses of NMDA receptors were higher as the timing of the release was delayed, reaching a $122 \%$ increase at $50 \mathrm{~ms}$

(Supplementary Fig. 4d-f). This increase is likely due to the binding of glutamate to those single-bound receptors, since this state can be maintained for tens of milliseconds and is favoured for activation by the second release. These results suggest that asynchronous release favours the activation of NMDA receptors in the absence of a $\mathrm{Mg}^{2+}$ block.

We next tested the effect of the AMPA receptor-mediated membrane depolarization on NMDA receptor activation in the presence of $\mathrm{Mg}^{2+}$. Two release events were induced simultaneously while the membrane potential was depolarized by 30 or $45 \mathrm{mV}$, mimicking the changes in membrane potential after a single release event near AMPA receptors or after simultaneous release at both AMPA and NMDA receptors, respectively (Fig. 3a). The constant voltage at resting potential was used as a control. $45 \mathrm{mV}$ depolarization doubled NMDA receptor activation compared to no membrane potential change (Fig. 3a,b). These results suggest that NMDA receptors can be efficiently activated by two release events (multivesicular release) 34,35 which are prevalent in these neurons ${ }^{4}$.

To test whether the timing of asynchronous release is important for NMDA receptor activation, we varied the timing of the second release by 5, 8, 10, and $20 \mathrm{~ms}$ (Fig. 3c). The kinetics of depolarization and repolarization following AMPA receptor activation are integrated into the platform ${ }^{13,15,36}$; the depolarization peaks between 3 to $5 \mathrm{~ms}$ after the release, increasing by $\sim 25 \mathrm{mV}$, and declines to $2 / 3$ of the maximum after $8 \mathrm{~ms}$ and almost to the resting potential by 10 ms (Supplementary Fig. 4g). The response from NMDA receptors peaked when 
asynchronous release occurred at $5 \mathrm{~ms}$ and decreased progressively as the asynchronous release was further delayed (Fig. 3d). These results are in sharp contrast to the activation of NMDA receptors in the absence of $\mathrm{Mg}^{2+}$, indicating that the kinetics of membrane depolarization and repolarization, and thereby $\mathrm{Mg}^{2+}$ unbinding and binding, determine the activity of NMDA receptors.

To test the importance of the location and order of release, we either flipped the order of release (first on NMDA receptors, and then on AMPA receptors) or applied both release at the same locations (Fig. 3e). The two release events were paired $5 \mathrm{~ms}$ apart to test the maximal response. Flipping the order did not change the response of NMDA receptors (Fig. 3f). However, when both release events were applied near NMDA receptors consecutively the response was 19\% lower (Fig. 3f), presumably because the membranes cannot be maximally depolarized. In fact, when we simulated with a higher level of depolarization, matching the degree of depolarization expected from releasing near AMPA receptors, the response of NMDA receptors was much stronger ( 32\% increase, Fig. 3f and Supplementary Fig. 4h-i). Together, these results suggest that the transsynaptic alignment of release sites and receptors ensures the maximal depolarization through AMPA receptors and thereby efficient activation of NMDA receptors.

In summary, we have revealed a novel spatial and temporal arrangement of release sites and receptors: synchronous and asynchronous release sites are aligned with AMPA and NMDA receptor clusters, respectively. Since the timing of asynchronous release coincides with the peak of postsynaptic membrane depolarization, the magnesium block on NMDA receptors is likely alleviated, allowing them to be activated by asynchronous release. However, it is not the order of release, but rather the activation of the AMPA receptors, that is essential for NMDA receptor 
activation. Thus, the recently described trans-synaptic alignment of the synchronous release sites with AMPA receptors ${ }^{14}$ likely ensures the proper activation of AMPA receptors, since their binding affinity for glutamate is very $\operatorname{low}^{32,33}$. A recent study suggests that neuroligin-1 may align release sites with AMPA receptors ${ }^{15}$. It is unknown how asynchronous release sites are aligned with NMDA receptors. Many synaptic adhesion molecules exist, and they all interact with the presynaptic release machinery as well as postsynaptic receptors and their scaffolding proteins $^{15,37-39}$. Thus, it is tempting to speculate that the arrangement of these molecules give rise to this unique trans-synaptic organization of release sites and receptors at the excitatory synapses. 


\section{Methods}

All animal procedures were performed according to the National Institute of Health guidelines and were approved by the Animal care and Use Committee at the Johns Hopkins University School of Medicine.

$\underline{\text { Animals }}$

Wild type mice (C57/BL6J) were purchased from Charles Laboratory and Gria2 ${ }^{(+-)}$mouse line was kindly provided by Dr. Richard Huganir. Gria $2^{(+/-)}$line was maintained as heterozygous and knock-out Gria2 $2^{(-/)}$pups at postnatal day 0 (P0) were used for neuronal culture.

\section{Neuron culture}

Both astrocytes and hippocampal neuron cultures were established from embryonic day 18 or P0 wild-type animals. Both sexes were indistinguishably used in this study. Astrocytes were harvested from cortices with trypsin treatment for $20 \mathrm{~min}$ at $37^{\circ} \mathrm{C}$ with shaking, followed by dissociation and seeding on T-75 flask. Astrocytes were grown in DMEM supplemented with $10 \%$ FBS and $0.1 \%$ penicillin-streptomycin at $37{ }^{\circ} \mathrm{C}$ and $5 \% \mathrm{CO} 2$ for $7-10$ days. Two clean 6 mm sapphire disks (Technotrade Inc) were placed per well of 12-well tissue culture plate and coated with poly-D-lysine ( $1 \mathrm{mg} / \mathrm{ml}$, Sigma) and collagen (ThermoFisher). Astrocytes serve as a feeder layer for neurons and were seeded (50,000/well) one week before hippocampal neuronal culture. On day 6, FUDR (80 uM) was added to inhibit cell division. On day 7, hippocampi were isolated and digested with papain $(20 \mathrm{U} / \mathrm{ml})$ for $30-60 \mathrm{~min}$ at $37{ }^{\circ} \mathrm{C}$ with shaking. An inactivation solution (2.5 mg of trypsin inhibitor and $0.5 \mathrm{mg}$ of albumin per mL of DMEM) was then applied for 5 min at $37^{\circ} \mathrm{C}$. Hippocampi were triturated by pipetting 4 x 20 times and cells were seeded on prepared astrocyte feeder layer with a density of 75,000/well and maintained in Neurobasal A 
media supplemented with B-27, Glutamax and $0.2 \%$ penicillin-streptomycin at $37{ }^{\circ} \mathrm{C}$ and $5 \%$ $\mathrm{CO}_{2}$. The cells were infected with lentivirus at days in vitro (DIV) 3-4 as needed and used for experiments on DIV 13-17.

\section{Expression constructs}

Lentiviral expression constructs were used to express transgenes in neurons. All vectors were based on the lentiviral shuttlevector FUGW ${ }^{40}$. Gria2 cDNA was N-terminally tagged with HaloTag and cloned in frame downstream of synapsin-1 promoter with NLS-GFP-P2A (NGP) sequence (a gift from Christian Rosenmund lab). The NLS-GFP signals were used to evaluate viral infection and intensity of transgene expression from this polycistronic construct. Nucleotides corresponding to hexa-histidine residues were inserted in Gria2 sequence downstream of the signal sequence using Gibson cloning strategy and NEB builder kit (New England Biolab) to generate NGP-(His)6-GluA2. To generate NGP-SnapTag::GluA2, the hexahistidine tag was replaced with a SnapTag by In-Fusion cloning (Takara Bio). pCI-SEP-NRI was a gift from Robert Malinow (Addgene plasmid \# 23999; http://n2t.net/addgene:23999 ;

RRID:Addgene_23999) ${ }^{41}$. Nucleotides corresponding to hexa-histidine were inserted downstream of the signal sequence of NR1 using Gibson cloning strategy and NEB builder kit (New England Biolab) followed by sub-cloning into the lentiviral shuttlevector FUGW to obtain NGP-(His)6-NR1. To generate NGP-SnapTag-NR1, the hexa-histidine tag was replaced with a SnapTag by In-Fusion cloning (Takara Bio).

$\underline{\text { Lentivirus production and infection }}$

Lentiviruses carrying the expression constructs were produced using the following procedures. The bottom surface of T-75 flasks was coated with poly-L-lysine $(0.2 \%$ in milliQ 
water). A day before the transfection, HEK293T cells were plated at $6.5 \times 10^{5} / \mathrm{ml}(10 \mathrm{ml}$ in T-75) in Neurobasal A (NBA) media containing $1 \%$ glutamax, $2 \%$ B27, and $0.2 \%$ penicillinstreptomycin. The shuttle vector $(\mathrm{FUGW})^{40}$ containing expression constructs and helper plasmids (VSV-G and CMV-dR8.9) were mixed at 20, 5, and $7.5 \mu \mathrm{g}$, respectively, in $640 \mu 1$ $\mathrm{NaCl}$ solution $(150 \mathrm{mM})$ (Solution I). Another solution (solution II) was prepared as follows: $246.7 \mu 1 \mathrm{H}_{2} \mathrm{O}, 320 \mu \mathrm{l} \mathrm{NaCl}(300 \mathrm{mM}), 73.3 \mu$ polyethylenimine $(0.6 \mu \mathrm{g} / \mu \mathrm{l})$. Solution I and II were mixed by vortexing and incubated at room temperature for 10 minutes followed by addition to the T-75 flask containing HEK293T cells. The cells were incubated at $37^{\circ} \mathrm{C}\left(5 \% \mathrm{CO}_{2}\right)$, and the viruses were harvested 3 days later. The media containing lentivirus was centrifuged at 4000 rpm to obtain 20-fold concentration using Amicon (Ultracel-100k). The infection efficiency was determined by infection in wild-type neurons that were separately prepared. For all the experiments, dissociated hippocampal neurons were infected on DIV 3-4 with lentiviruses carrying the expression constructs. The infection rate of $95 \%$ was achieved in all cases.

\section{Ni-NTA-gold labelling}

For gold labelling, solution containing Ni-NTA-gold (Nanoprobes) was added to each well containing two sapphire disks so that the final concentration of $5 \mathrm{nM}$ is achieved. Cells were incubated for $30 \mathrm{~min}$ in the $\mathrm{CO}_{2}$ incubator set at $37^{\circ} \mathrm{C}$. Each disk was washed thoroughly by agitating in a small petri dish $(30 \mathrm{~mm})$ containing physiological saline solution $(140 \mathrm{mM} \mathrm{NaCl}$, $2.4 \mathrm{mM} \mathrm{KCl}, \mathrm{CaCl}_{2} 1.2 \mathrm{mM}$, and $3.8 \mathrm{mM} \mathrm{MgCl}_{2}, 10 \mathrm{mM}$ HEPES, $10 \mathrm{mM}$ glucose; $\mathrm{pH}$ adjusted to 7.3 with $\mathrm{NaOH}, 300 \pm 5 \mathrm{mOsm})$. The washing procedure was repeated three times to minimize background labelling. Immediately after washing, cells were mounted for highpressure freezing. 


\section{High-pressure freezing and freeze-substitution}

Cells cultured on sapphire disks were frozen using a high pressure freezer (EM ICE, Leica Microsystems). Following gold labelling, each disk with neurons was transferred into the physiological saline solution containing NBQX (3 $\mu \mathrm{M}$, Tocris) and bicuculine (30 $\mu \mathrm{M}$; Tocris), which were added to block recurrent synaptic activity during the zap-and-freeze experiments. The disk was mounted onto the photoelectric middle plate with neurons facing up. A $100 \mu \mathrm{m}$ spacer ring was placed on top of the sapphire disk. Then, another blank sapphire disk was placed on top of the spacer ring to make a "sandwich". Finally, a rubber ring was put on top of the "sandwich" to hold it in place. The entire assembled middle plate was then placed on a piece of filter paper to remove the excess liquid, loaded between two half cylinders, and transferred into the freezing chamber. An electrical field of $10 \mathrm{~V} / \mathrm{cm}$ was applied for $1 \mathrm{~ms}$ to induce a single action potential, and cells were frozen $5 \mathrm{~ms}$ and $11 \mathrm{~ms}$ after the stimulus ${ }^{4}$. For no stimulation control, the photoelectric middle plate was programmed not to discharge. The frozen sample was automatically dropped into a storage dewar filled with liquid nitrogen.

After freezing, the middle plate with sapphire disks were transferred to a cup containing anhydrous acetone $\left(-90^{\circ} \mathrm{C}\right)$, which was placed in an automated freeze substitution system (EM AFS2, Leica microsystems) using pre-chilled tweezers. The cryovials containing fixative (1\% glutaraldehyde, $1 \%$ osmium tetroxide and 1\% water in anhydrous acetone) were stored in liquid nitrogen and moved to AFS2 before use. After disassembling the freezing apparatus, sapphire disks with neurons were transferred into cryovials in the AFS, which is set at $-90{ }^{\circ} \mathrm{C}$, using prechilled tweezers. The freeze substitution program was as follows: $-90{ }^{\circ} \mathrm{C}$ for $6-10$ hours, $-90{ }^{\circ} \mathrm{C}$ to $-20{ }^{\circ} \mathrm{C}$ in 14 hours, $-20^{\circ} \mathrm{C}$ for 12 hours, and $-20{ }^{\circ} \mathrm{C}$ to $20{ }^{\circ} \mathrm{C}$ in 4 hours. 


\section{Embedding, sectioning and transmission electron microscopy}

Following freeze-substitution, fixatives were washed with anhydrous acetone for three times, 10 min each. After washing, samples were infiltrated through $30 \%, 70 \%$ and $90 \%$ eponaraldite in anhydrous acetone every two hours. Then samples were transferred to caps of polyethylene BEEM capsules with $90 \%$ epon araldite and incubate overnight at $4{ }^{\circ} \mathrm{C}$. Next day, samples were incubated in the caps of polyethylene BEEM capsules with $100 \%$ epon araldite (epon $6.2 \mathrm{~g}$; araldite $4.4 \mathrm{~g}$; DDSA $12.2 \mathrm{~g}$, and BDMA $0.8 \mathrm{ml}$ ) at room temperature. Samples were transferred to new caps with fresh $100 \%$ epon araldite every 2 hours three times, after which samples were baked at $60^{\circ} \mathrm{C}$ for 48 hours.

After resin was cured, sapphire disks were removed from resin. Cells were embedded in the resin block. Then, the block was cut into small pieces and place atop of a dummy block using super glue for sectioning. $40 \mathrm{~nm}$ sections were cut using an ultramicrotome (EM UC7, Leica microsystems) and collected on single-slot copper grids coated with $0.7 \%$ pioloform. The sections were stained with $2.5 \%$ uranyl acetate in $75 \%$ methanol and then imaged at $80 \mathrm{kV}$ at the 93,000x magnification on a Philips CM120 transmission electron microscope equipped with an AMT XR80 camera. About 100 electron micrographs per sample were taken blindly. To avoid the sampling bias, synapses were found by bidirectional raster scanning along the section at 93,000x. Synapses were identified by a vesicle-filled presynaptic bouton and a postsynaptic density. 


\section{$\underline{\text { Spin-mill serial block face imaging }}$}

Samples were sent to ThermoFisher for spin-mill serial block face imaging. Spin-milling experiments were performed on a DaulBeam instrument (Thermo Fisher Scientific, Helios Hydra). A whole resin block (6 mm diameter) was glued onto a scanning electron microscope (SEM) stub using silver conductive epoxy without pre-trimming or sputter coating. The sample was positioned at the eucentric position of the system, and the stage was tilted to -34 degrees, such that the focused-ion beam (FIB) angle of incidence was 4 degrees from glancing relative to the sample surface.

The spin-milling process consists of the following sequence. First, oxygen FIB beam (12 $\mathrm{keV}, 65 \mathrm{nA}$ ) was applied in a $400 \mu \mathrm{m} \times 100 \mu \mathrm{m}$ box pattern on desired sample area for $10 \mathrm{~s}$ (dwell time set at $200 \mathrm{~ns}$ ) to expose a new surface of the sample. The stage was compucentrically rotated 60 degrees, and the sample milled again with another FIB exposure. This process was repeated 6 times to achieve a full 360-degree rotation of the sample. Ion flux was delivered to the sample from several different azimuthal directions to reduce textural artifacts generated during the ion milling. One full rotation of milling constituted a "z slice". Second, the sample was tilted back to a stage tilt of zero degree to perform SEM imaging. Images were collected from multiple regions-of-interest. These two steps are automated with Autoscript software (Thermo Fisher Scientific) and repeated until the desired volume of images are collected - similar to the serial block face imaging technique ${ }^{42}$. The milling slice thickness was controlled to achieve $20 \mathrm{~nm}$. Ten areas of interests were acquired in parallel with a X, Y pixel size of $1 \mathrm{~nm}$. A total of $\sim 40$ slices was collected from each sample. The resulting 3-D dataset were aligned and visualized using the Amira software (Thermo Fisher Scientific). 


\section{Electron microscopy image analysis}

All the images from a single experiment were shuffled for analysis as a single pool using a custom R (R Development Team) script. Images that could not be reliably segmented, either because the image was not of a bona fide synapse or morphology was too poor, were excluded from segmentation; this was done only after randomizing the images. No other data were excluded. The plasma membrane, active zone, postsynaptic density, docked synaptic vesicles, synaptic vesicles close to the active zone, pits (putative fusion events), and gold particles were annotated in ImageJ using a custom macro. The active zone was identified as the region of the presynaptic plasma membrane juxtaposed to the postsynaptic density. Docked vesicles were identified by their membrane appearing to be in contact with the plasma membrane at the active zone ( $0 \mathrm{~nm}$ from the plasma membrane), that is, there are no lighter pixels between the membranes. Pits were identified as smooth curvature (not mirrored by the postsynaptic membrane) in an otherwise straight membrane. These pits are considered exocytic ${ }^{4}$. Pits outside the active zone are considered endocytic or membrane ruffles, as this is the primary site for ultrafast endocytosis ${ }^{30}$. Under these criteria, we could miss or over-annotate vesicles and pits. To minimize the bias and maintain consistency, all image segmentation, still in the form of randomized files, was thoroughly checked by a second member of the lab. However, no corrections were made for synaptic vesicles since vesicles are much more abundant and the same criteria were used to annotate them in all conditions. A similar amount of overestimate is expected in this case. Features were then quantitated using custom MATLAB (MathWorks) scripts.

Location of pits, docked vesicles, and gold particles within the active zone/postsynaptic density from single sections was calculated from the distance from the center of the pit to the 
center and the edge of the active zone in 2D. Distance from the center was normalized by dividing the distance to the edge by the half-width of the active zone. For 3D data, the distance to the center of the active zone was calculated from serial sections. First, the location in 2D was calculated as above. Then, the $3 \mathrm{D}$ distance was calculated to the center of the active zone in the middle section of the series using the Pythagorean theorem with assumption that each section is the same thickness and the center of the active zone aligns in each image. Locations in 3D data were further corrected to be the density of vesicles/pits at each distance from the center of the active zone or postsynaptic density. To calculate density of vesicles, pits, and gold particles from the center to the edge in $3 \mathrm{D}$ reconstructions, the radial position of each vesicle/pit/gold particle was converted to the fractional area of a circle bounded by that radius. In the case of a unit circle (distance from center to edge is by definition 1 when data normalized to the size of the postsynaptic density), this is simply the square of the original normalized distance to the center. Example micrographs shown were adjusted in brightness and contrast to different degrees (depending on the varying brightness and contrast of the raw images), rotated, and cropped in Adobe Photoshop.

\section{Cluster analysis}

Sequential annotated electron microscopy slices of gold-labelled NMDA and AMPA receptors were used to generate a 3-D spatial map of receptors for each synapse (MATLAB 2019b, Mathworks). For each synapse, K-means clustering (Lloyd's algorithm) was performed for 1 to $\mathrm{N}$ number clusters, where $\mathrm{N}$ is the total number of gold-labelled receptors. The optimal number of clusters was obtained by calculating the knee-point of the within-cluster sum of square differences (SSD) as a function of number of clusters. A final generated synapse with the 
optimized number of clusters was then rendered over each synapse for both AMPA and NMDA receptors. These spatial maps allow visualization and measurement of the locations of each receptor with respect to the center of the synapse. To determine whether the clustering of these receptors was due to chance, for each mapped synapse and respective number of particles, we generated 50 maps with randomized particle positions using custom scripts. The above K-means clustering paradigm was then run on all 50 maps and the mean SSD was recorded for each synapse. 2D scatter plots of SSD and synaptic sizes for the experimental and randomized condition for AMPA and NMDA receptors were generated, where low SSD indicates more tightly packed clusters. Linear regression analysis (Prism 8.2.0, GraphPad) was run for each set of conditions to determine whether the experimentally-clustered receptors were significantly different than randomized clusters.

\section{Computer simulations}

Computer modeling was performed using the MCell/CellBlender simulation environment (mcell.org) version 3.5.0-9 for Linux. The realistic model of glutamatergic synaptic environment was constructed from 3-D electron microscopy of hippocampal area CA1 neuropil as previously described $^{13,43,44}$. The AMPAR chemical kinetic properties were obtained from the well-established model published ${ }^{32}$, and the kinetic parameters were adjusted to fit with the recorded mEPSCs ${ }^{15}$. The NMDAR kinetics were obtained from Vargas-Caballero and Robinson ${ }^{17}$. Since the time course of the diffusion and presence of glutamate in the synaptic cleft is especially important in the model presented here, our model also included realistic extracellular space a kinetic model of glutamate transporters distributed on astrocytic glial processes in the surrounding neuropil as previously described ${ }^{13}$. 
The initial distribution of AMPA and NMDA receptors as well as the location of the presynaptic neurotransmitter release site was established by running a dynamic simulation to allow self-organization of the distributions. To accomplish this, two surface properties were defined: the synapse and the postsynaptic density (identified on electron microscopy data). According to the literature 200 PSD-95 molecules, 60 AMPA receptors, and 30 NMDA receptors were available on the spine head. These molecules were allowed to freely diffuse at the synapse. Inside the postsynaptic density, PSD-95 was reversibly palmitoylated (pPSD-95) at a defined rate (kon=35, koff=0.7).

A clusterization point called "L" was placed at the center of the postsynaptic density. pPSD-95 aggregates in contact with $\mathrm{L}(\mathrm{kon}=7$, $\mathrm{koff}=1)$ to form a domain. Mobile NMDA receptors interacted with this domain and were trapped into an NMDA receptor cluster (kon $=10$, koff = 1). A mobile "G" molecule was released inside the postsynaptic density and was immobilized at random location when it randomly interacted with a pPSD-95. After immobilization, the molecule of "G" recruited the insertion of a pre-synaptic neurotransmitter release site into the pre-synaptic membrane at the point closest to the location of G. At the same time, "G" clustered pPSD-95 $(\mathrm{kon}=100, \mathrm{koff}=1)$ which, in turn, clustered AMPA receptor (kon $=10$, koff $=1)$.

The approach to a final steady-state organization of AMPA receptor, and NMDA receptor at the synapse was simulated with a time step of $1 \mathrm{~ms}$ for 10,000 iterations (10 s), until reaching a steady. It is important to note that the means employed here to achieve receptor organization is only intended to give the desired final organization in our model and is not intended to model the physiological mechanisms by which this occurs in real synapses. After reaching the desired organization, the simulations were switched to a time step of $1 \mu$ s for 250,000 iterations to model 
the AMPA receptor and NMDA receptor and when the glutamate was released at the pre-synaptic level, in front of the "G" aligned with AMPA receptor or in front of "L" aligned with the NMDA receptor.

After binding neurotransmitter, the flux of ionic current through activated NMDAR is voltage dependent due to channel blockade by $\mathrm{Mg}^{2+}$ at hyperpolarized membrane potential ${ }^{18}$. We simulated this voltage-dependent blockade by a method described previously ${ }^{13}$. Briefly, the neural simulation program NEURON was used to simulate excitatory postsynaptic potentials of the desired timing and amplitude in the spine head located on the dendritic branch of a modeled pyramidal neuron. The time-varying voltages recorded at the spine during these stimuli were used to drive voltage-dependent transition rates in the model of NMDA receptor activation kinetics. The voltage changes alter the kinetic constants for relief of the $\mathrm{Mg}^{2+}$ block of the NMDA receptors and determine the ion current flux through open NMDA receptors. Each individual NMDA receptor channel opens and fluxes current in the simulation only when glutamate is bound to the receptor at the time that the $\mathrm{Mg}^{2+}$ block is relieved. MCell uses stochastic Monte Carlo methods and simulation results reflect the realistic behavior of stochastic channel fluctuations. For all computational experiments, 48 trials were performed allowing estimation of the mean and standard deviation of the time course of channel activation.

\section{$\underline{\text { Statistical analysis }}$}

All data showing distribution of receptors, vesicles, and pits are pooled from multiple experiments. The number data shown are per experiment. All data were initially examined on a per-experiment basis (with all freezing done on the same day and all segmentation done in a single randomized batch); none of the pooled data show any result that was not found in each 
replicate individually. We did not predetermine sample sizes using power analysis, but based them $(\mathrm{N}=2-3, \mathrm{n}>200)$ on our prior experience with flash-and-freeze data ${ }^{30,31,45}$. An alpha of 0.05 was used for statistical hypothesis testing. All data were tested for normality by D’Agostino-Pearson omnibus test to determine whether parametric or nonparametric methods should be used. Comparisons between two groups were performed using a two-tailed Welch twosample t-test or Wilcoxon rank-sum test. Comparisons between multiple groups followed by full pairwise comparisons were performed using one-way analysis of variance (ANOVA) followed by Tukey's HSD test or Kruskal-Wallis test followed by Dunn's multiple comparisons test. For testing whether locations of pits or receptors were biased toward the center or edge of the synapse, a two-tailed one-sample t-test or Wilcoxon rank-sum test with a theoretical median of 0.5 was used (each of these p-values, as well as that of the comparisons between pit locations in different samples, were accordingly corrected for multiplicity using Bonferroni's method). All statistical analyses were performed and all graphs created in Graphpad Prism.

\section{Life Sciences Reporting Summary}

More details on experimental procedures, materials, and statistics are available in the Life Sciences Reporting Summary.

Data and code availability

The data underlying this work, as well as custom R and MATLAB scripts, are available upon request. 


\section{Acknowledgements}

We are indebted to Quan Gan, Kie Imoto, and Chengxiu Zhang for cell culture, help with gold labelling experiments, and stimulating discussions. We also thank Mike Delanoy and Barbara Smith for technical assistance with electron microscopy. We thank Cveta Tomova, Chad Rue and Kenny Mani at Thermo Fisher Scientific for facilitating the collaboration on Helios Hydra DualBeam, and Christian Rosenmund and Marisa Brockmann for sharing unpublished data. We also thank the Marine Biological Laboratory and their Neurobiology course for supporting the initial set of experiments. S.W. and this work were supported by start-up funds from the Johns Hopkins University School of Medicine, Johns Hopkins Discovery funds, and the National Science Foundation (1727260), the National Institutes of Health (1DP2 NS111133-01 and 1R01 NS105810-01A1) awarded to S.W. S.W. is an Alfred P. Sloan fellow, McKnight Foundation Scholar, and Klingenstein and Simons Foundation scholar. G.F.K. was supported by a grant from the National Institutes of Health to the Biochemistry, Cellular and Molecular Biology program of the Johns Hopkins University School of Medicine (T32 GM007445) and is a National Science Foundation Graduate Research Fellow (2016217537). E.H. and T.B. are supported by CRCNSNIH-ANR grant AMPAR-T. The EM ICE high-pressure freezer was purchased partly with funds from an equipment grant from the National Institutes of Health (S10RR026445) awarded to Scot C Kuo.

\section{Author Contributions}

S.W. and S.L. designed the experiments and analyzed the data, and wrote the manuscript. S.L., S.R., and G.F.K. performed all freezing experiments and single-section electron microscopy sample preparation, imaging, and analysis, with technical assistance from S.W. J.W. performed the spin-mill imaging, and S.L., S.A.L., C.P., and S.S., analyzed the 3-D data. S.W. and S.A.L. 
developed MATLAB code for image analysis. H.F. and R.R. performed pilot experiments and electron microscopy sample preparation, imaging, and analysis. M.W.D. designed the prototype zap-and-freeze stimulation device. S.W. T.B. and E.H. developed and performed computer simulations. S.W. funded and oversaw the research. 


\section{References}

1. Kaeser, P. S. \& Regehr, W. G. Molecular mechanisms for synchronous, asynchronous, and spontaneous neurotransmitter release. Annu. Rev. Physiol. 76, 333-363 (2014).

2. Heuser, J. \& Reese, T. Synaptic-Vesicle Exocytosis Captured by Quick-Freezing. in Fourth Intensive Study Program in the Neuroscience 573-600 (M.I.T. press, 1979).

3. Kaeser, P. S. \& Regehr, W. G. The readily releasable pool of synaptic vesicles. Curr. Opin. Neurobiol. 43, 63-70 (2017).

4. Synaptic vesicles undock and then transiently dock after an action potential | bioRxiv. https://www.biorxiv.org/content/10.1101/509216v1.

5. Bekkers, J. M. \& Stevens, C. F. NMDA and non-NMDA receptors are co-localized at individual excitatory synapses in cultured rat hippocampus. Nature 341, 230-233 (1989).

6. Dingledine, R., Borges, K., Bowie, D. \& Traynelis, S. F. The glutamate receptor ion channels. Pharmacol. Rev. 51, 7-61 (1999).

7. Tang, C. M., Dichter, M. \& Morad, M. Quisqualate activates a rapidly inactivating high conductance ionic channel in hippocampal neurons. Science 243, 1474-1477 (1989).

8. Trussell, L. O. \& Fischbach, G. D. Glutamate receptor desensitization and its role in synaptic transmission. Neuron 3, 209-218 (1989).

9. Patneau, D. K. \& Mayer, M. L. Structure-activity relationships for amino acid transmitter candidates acting at N-methyl-D-aspartate and quisqualate receptors. J. Neurosci. Off. J. Soc. Neurosci. 10, 2385-2399 (1990).

10. Erreger, K., Dravid, S. M., Banke, T. G., Wyllie, D. J. A. \& Traynelis, S. F. Subunit-specific gating controls rat NR1/NR2A and NR1/NR2B NMDA channel kinetics and synaptic signalling profiles. J. Physiol. 563, 345-358 (2005). 
11. Uteshev, V. V.\& Pennefather, P. S. A mathematical description of miniature postsynaptic current generation at central nervous system synapses. Biophys. J. 71, 1256-1266 (1996).

12. Zheng, K. et al. Nanoscale diffusion in the synaptic cleft and beyond measured with timeresolved fluorescence anisotropy imaging. Sci. Rep. 7, 1-10 (2017).

13. Bartol, T. M. et al. Computational reconstitution of spine calcium transients from individual proteins. Front. Synaptic Neurosci. 7, (2015).

14. Tang, A.-H. et al. A transsynaptic nanocolumn aligns neurotransmitter release to receptors. Nature 536, 210-214 (2016).

15. Haas, K. T. et al. Pre-post synaptic alignment through neuroligin-1 tunes synaptic transmission efficiency. eLife 7, e31755 (2018).

16. Nahum-Levy, R., Lipinski, D., Shavit, S. \& Benveniste, M. Desensitization of NMDA receptor channels is modulated by glutamate agonists. Biophys. J. 80, 2152-2166 (2001).

17. Vargas-Caballero, M. \& Robinson, H. P. C. Fast and Slow Voltage-Dependent Dynamics of Magnesium Block in the NMDA Receptor: The Asymmetric Trapping Block Model. $J$. Neurosci. 24, 6171-6180 (2004).

18. Nowak, L., Bregestovski, P., Ascher, P., Herbet, A. \& Prochiantz, A. Magnesium gates glutamate-activated channels in mouse central neurones. Nature 307, 462-465 (1984).

19. Mayer, M. L., Westbrook, G. L. \& Guthrie, P. B. Voltage-dependent block by Mg2+ of NMDA responses in spinal cord neurones. Nature 309, 261-263 (1984).

20. Seeburg, P. H. et al. The NMDA receptor channel: molecular design of a coincidence detector. Recent Prog. Horm. Res. 50, 19-34 (1995).

21. Malinow, R. \& Miller, J. P. Postsynaptic hyperpolarization during conditioning reversibly blocks induction of long-term potentiation. Nature 320, 529-530 (1986). 
22. Kauer, J. A., Malenka, R. C. \& Nicoll, R. A. A persistent postsynaptic modification mediates long-term potentiation in the hippocampus. Neuron 1, 911-917 (1988).

23. Bi, G. Q. \& Poo, M. M. Synaptic modifications in cultured hippocampal neurons: dependence on spike timing, synaptic strength, and postsynaptic cell type. J. Neurosci. Off. J. Soc. Neurosci. 18, 10464-10472 (1998).

24. Hainfeld, J. F., Liu, W., Halsey, C. M. R., Freimuth, P. \& Powell, R. D. Ni-NTA-Gold Clusters Target His-Tagged Proteins. J. Struct. Biol. 127, 185-198 (1999).

25. Los, G. V. et al. HaloTag: A Novel Protein Labeling Technology for Cell Imaging and Protein Analysis. ACS Chem. Biol. 3, 373-382 (2008).

26. Oxygen plasma focused ion beam scanning electron microscopy for biological samples | bioRxiv. https://www.biorxiv.org/content/10.1101/457820v1.

27. Nair, D. et al. Super-Resolution Imaging Reveals That AMPA Receptors Inside Synapses Are Dynamically Organized in Nanodomains Regulated by PSD95. J. Neurosci. 33, 1320413224 (2013).

28. Masugi-Tokita, M. et al. Number and Density of AMPA Receptors in Individual Synapses in the Rat Cerebellum as Revealed by SDS-Digested Freeze-Fracture Replica Labeling. $J$. Neurosci. 27, 2135-2144 (2007).

29. Nusser, Z. et al. Cell Type and Pathway Dependence of Synaptic AMPA Receptor Number and Variability in the Hippocampus. Neuron 21, 545-559 (1998).

30. Watanabe, S. et al. Ultrafast endocytosis at Caenorhabditis elegans neuromuscular junctions. eLife 2, e00723 (2013).

31. Watanabe, S. et al. Ultrafast endocytosis at mouse hippocampal synapses. Nature 504, 242$247(2013)$. 
32. Jonas, P., Major, G. \& Sakmann, B. Quantal components of unitary EPSCs at the mossy fibre synapse on CA3 pyramidal cells of rat hippocampus. J. Physiol. 472, 615-663 (1993).

33. Colquhoun, D., Jonas, P. \& Sakmann, B. Action of brief pulses of glutamate on AMPA/kainate receptors in patches from different neurones of rat hippocampal slices. $J$. Physiol. 458, 261-287 (1992).

34. Tong, G. \& Jahr, C. E. Multivesicular release from excitatory synapses of cultured hippocampal neurons. Neuron 12, 51-59 (1994).

35. Rudolph, S., Tsai, M.-C., von Gersdorff, H. \& Wadiche, J. I. The ubiquitous nature of multivesicular release. Trends Neurosci. 38, 428-438 (2015).

36. Mainen, Z. F., Joerges, J., Huguenard, J. R. \& Sejnowski, T. J. A model of spike initiation in neocortical pyramidal neurons. Neuron 15, 1427-1439 (1995).

37. Jang, S., Lee, H. \& Kim, E. Synaptic adhesion molecules and excitatory synaptic transmission. Curr. Opin. Neurobiol. 45, 45-50 (2017).

38. Williams, M. E., de Wit, J. \& Ghosh, A. Molecular Mechanisms of Synaptic Specificity in Developing Neural Circuits. Neuron 68, 9-18 (2010).

39. Biederer, T., Kaeser, P. S. \& Blanpied, T. A. Transcellular Nanoalignment of Synaptic Function. Neuron 96, 680-696 (2017).

40. Lois, C., Hong, E. J., Pease, S., Brown, E. J. \& Baltimore, D. Germline transmission and tissue-specific expression of transgenes delivered by lentiviral vectors. Science 295, 868-872 (2002).

41. Kopec, C. D., Li, B., Wei, W., Boehm, J. \& Malinow, R. Glutamate receptor exocytosis and spine enlargement during chemically induced long-term potentiation. J. Neurosci. Off. J. Soc. Neurosci. 26, 2000-2009 (2006). 
42. Denk, W. \& Horstmann, H. Serial block-face scanning electron microscopy to reconstruct three-dimensional tissue nanostructure. PLoS Biol. 2, e329 (2004).

43. Bartol, T. M., Jr et al. Nanoconnectomic upper bound on the variability of synaptic plasticity. eLife 4, e10778 (2015).

44. Kinney, J. P. et al. Extracellular sheets and tunnels modulate glutamate diffusion in hippocampal neuropil. J. Comp. Neurol. 521, 448-464 (2013).

45. Watanabe, S. et al. Clathrin regenerates synaptic vesicles from endosomes. Nature 515, 228233 (2014). 
Figure biokxivpreprint doi! https://doi.org/10.1101/2020.05.01.072157; this version posted May 2, 2020. The copyright holder for this preprint (which was not certified by peer review) is the author/funder. All rights reserved. No reuse allowed without permission.

a

His-tag::GluA2

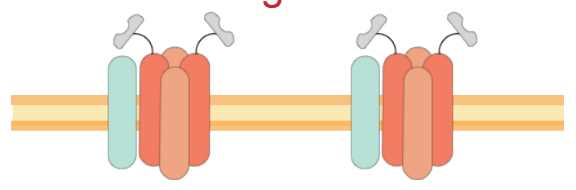

Ni-NTA-Au
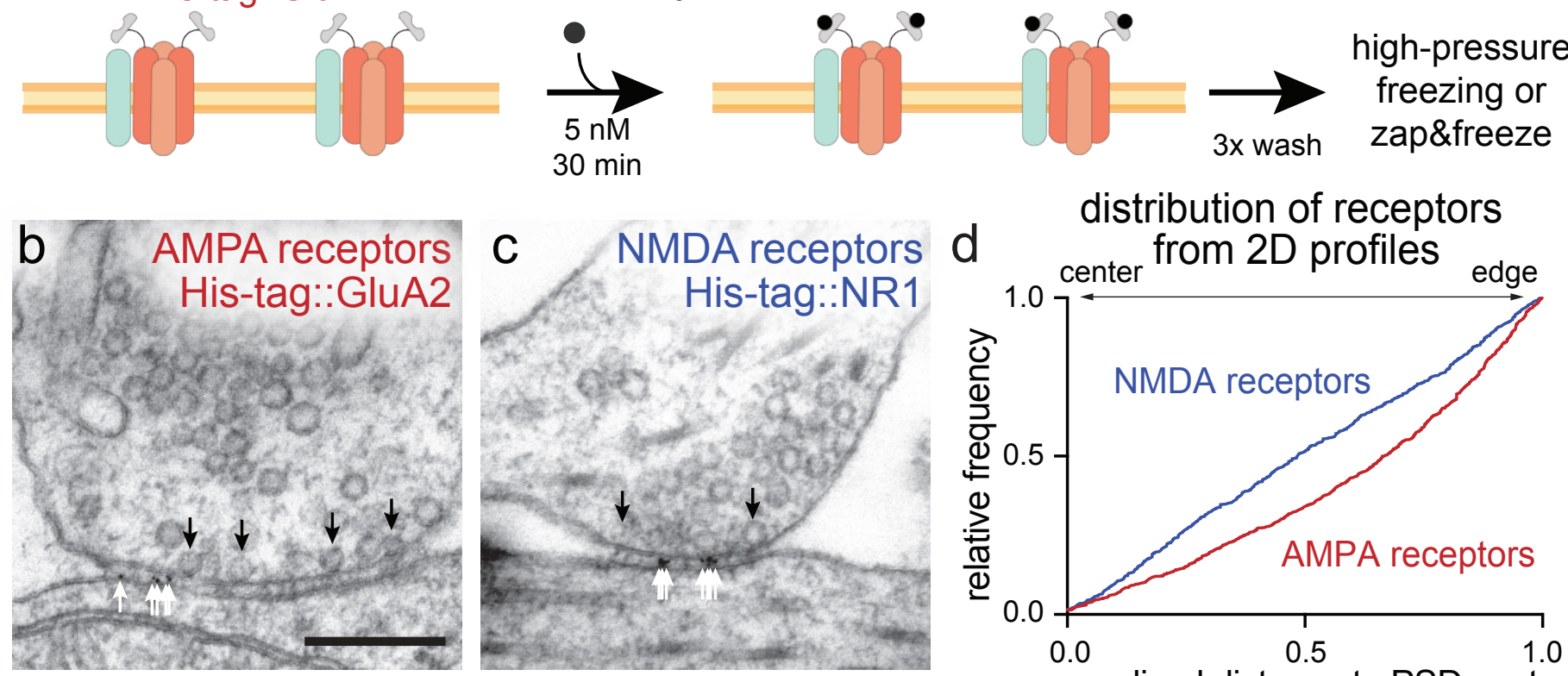

e

3D reconstructions

\section{AMPA receptors}

NMDA receptors
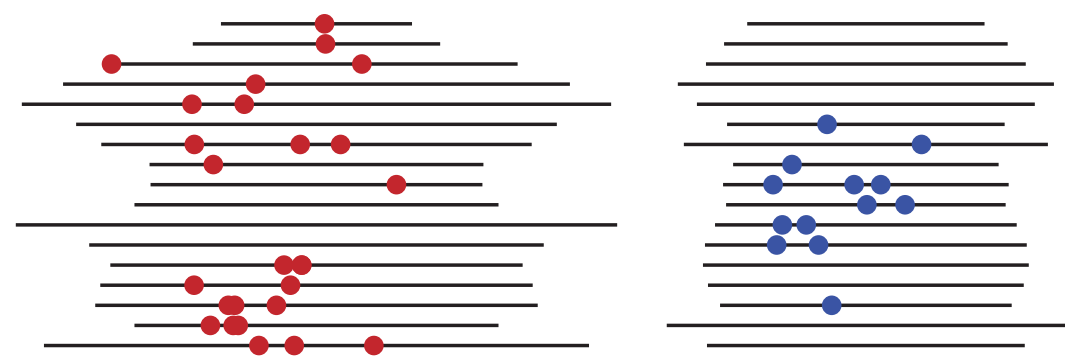

$50 \mathrm{~nm}$

g

K-means cluster analysis

AMPA receptors

NMDA receptors
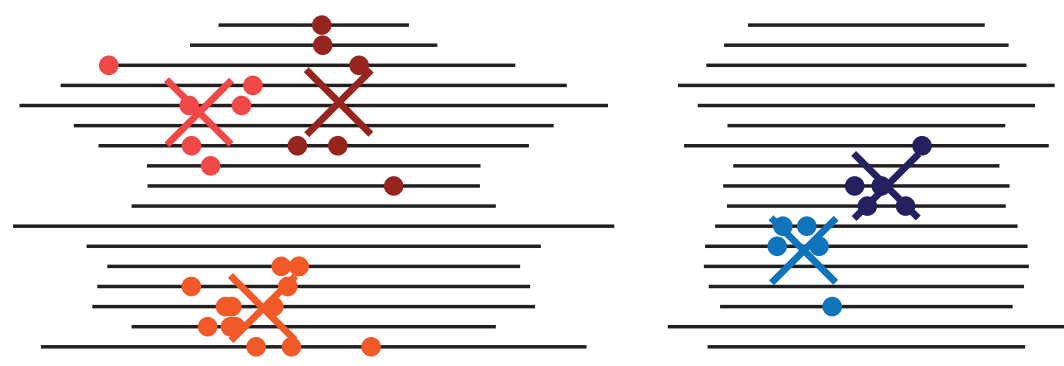

j

sum of squared differences

AMPA receptors

NMDA receptors
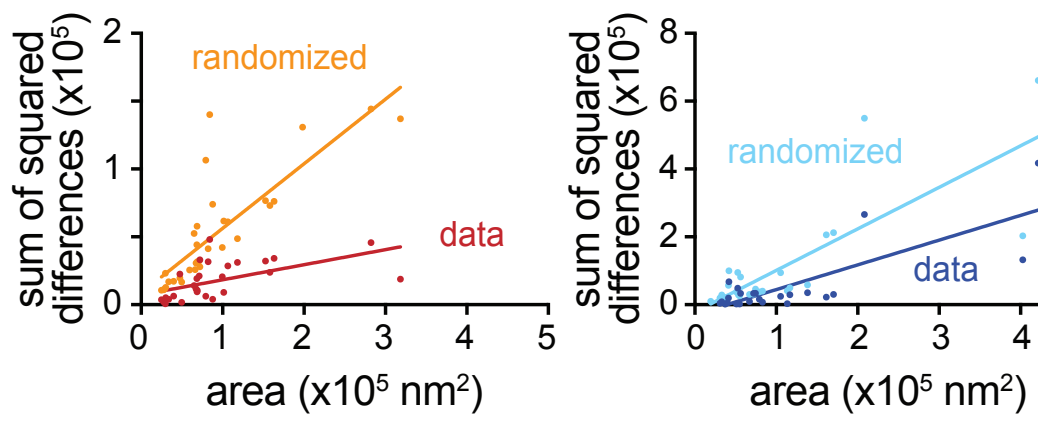

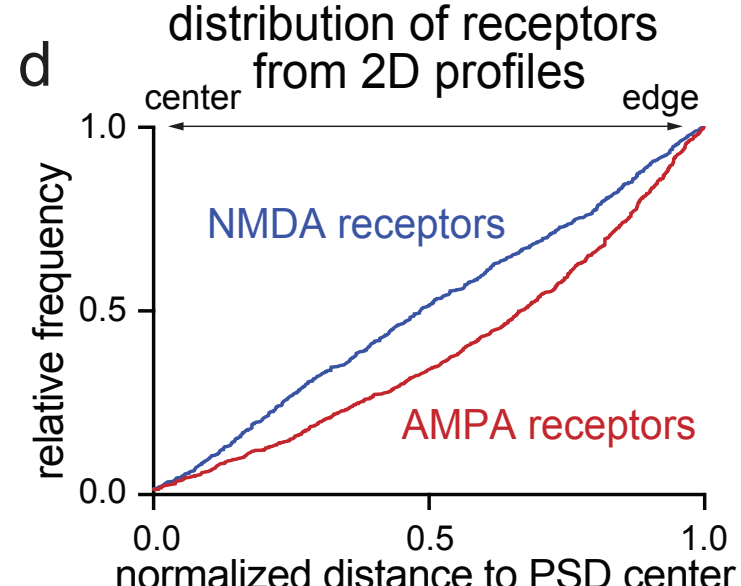

f

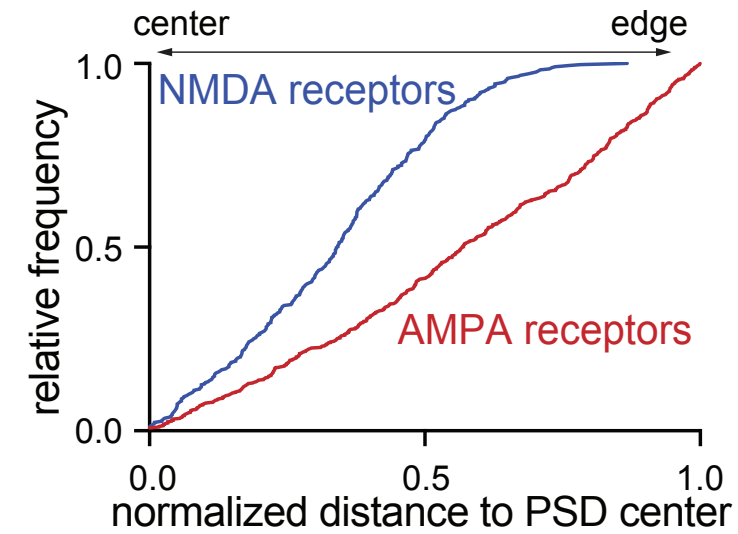

h area of the i \# clusters reconstructed PSD per synapse
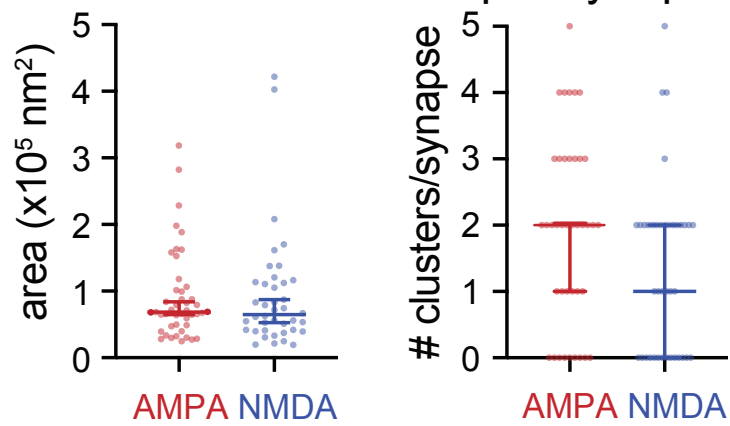

k \# gold particles I \# gold particles per synapse

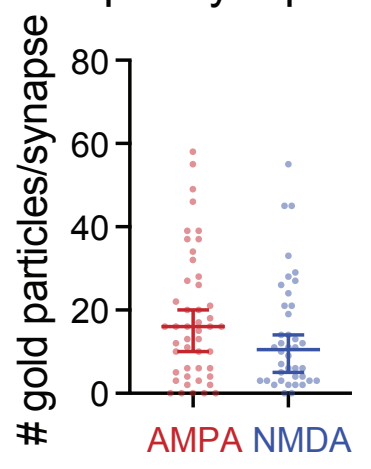

per synapse

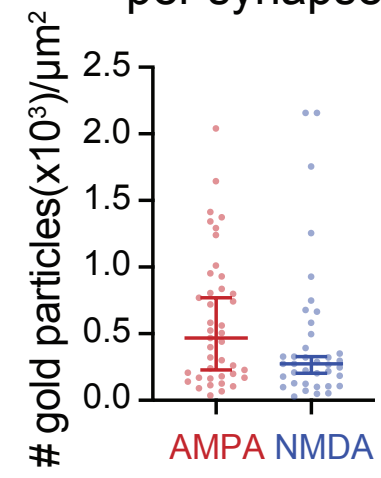




\section{Figure Legends}

Fig. 1. AMPA receptors and NMDA receptors cluster at the periphery and the center of the postsynaptic density, respectively

a, Schematic of the small metal affinity staining of His-tag (SMASH) strategy for live-cell labelling of overexpressed His-tagged surface GluA2 (AMPA receptors) with nickel-NTA-gold $(5 \mathrm{~nm})$. b-c, Example transmission electron micrographs of synapses after SMASH labelling and high-pressure freezing, showing gold particles in the synaptic cleft of wild-type neurons expressing His-tag::GluA2 (b) and His-tag::NR1 (c). Black arrows: docked synaptic vesicles. White arrows: gold particles. d, Cumulative relative frequency distribution of lateral distances from gold particles to the center of the postsynaptic density (PSD) from 2-D profiles. Distances are normalized to the length of the PSD: a gold particle at 0 would be exactly at the center and at 1 exactly at the edge. AMPA receptors are biased towards the edge (median $=0.7, \mathrm{p}<0.001, \mathrm{n}=$ 891 particles, $\mathrm{N}=4$ cultures), while NMDA receptors are uniformly distributed (median $=0.5, \mathrm{p}$ $>0.8, \mathrm{n}=746$ particles, $\mathrm{N}=4$ cultures). e, Examples of synapses from spin-mill serial block face scanning electron microscopy; each line indicates the extent of the cleft in a single $20 \mathrm{~nm}$-thick 2-D profile, each circle indicates the location of a gold particle. $\mathbf{f}$, Cumulative relative frequency distribution of lateral distances from gold particles to the center of the postsynaptic density (PSD) from 3-D profiles. Distances are normalized to the size of the PSD and corrected as fractional area assuming a circular PSD: a gold particle at 0 would be exactly at the center, at 1 exactly at the edge, and at 0.25 equidistant between center and edge. AMPA receptors are slightly biased towards the edge (median $=0.6, \mathrm{p}<0.001, \mathrm{n}=840$ particles, $\mathrm{N}=2$ cultures), while NMDA receptors are biased towards the center (median $=0.3, \mathrm{p}<0.001, \mathrm{n}=550$ particles, $\mathrm{N}=2$ cultures). $\mathbf{g}$, Same as $\mathbf{e}$, except showing the centers of clusters determined by k-means 
clustering. h, Areas of the reconstructed postsynaptic densities. Each dot: a single reconstructed synapse. Error bars: median and 95\% confidence interval, $\mathrm{p}>0.6$, Mann-Whitney test. i, Number of clusters per synapse determined by k-means clustering. Each dot: a single reconstructed synapse. Error bars: median and 95\% confidence interval, $\mathrm{p}=0.06$, Mann-Whitney test. j, Sum of squared differences, calculated from each particle to the centroid of the cluster. Data: the actual distances of gold particles to their cluster center. Randomized: the distances of gold particles to their putative cluster center after randomizing the locations of gold particles at each synapse. Each dot: a synapse. Simple linear regression test: AMPA receptors, data, $\mathrm{R}^{2}=0.32$, randomized $=0.67, \mathrm{p}<0.001$; NDA receptors, data, $\mathrm{R}^{2}=0.63$, randomized $=0.62, \mathrm{p}=0.04$. $\mathbf{k}$ I, Number of gold particles per synapse (k) and per $\mu \mathrm{m}^{2}$ (l). Bias of particle locations toward the center or edge of the postsynaptic density in $\mathbf{d}$ and $\mathbf{f}$ was tested by comparing each group to a theoretical median of 0.5 using one-sample two-tailed Wilcoxon signed-rank tests. See

Supplementary Data Table 1 for full pairwise comparisons and summary statistics. 

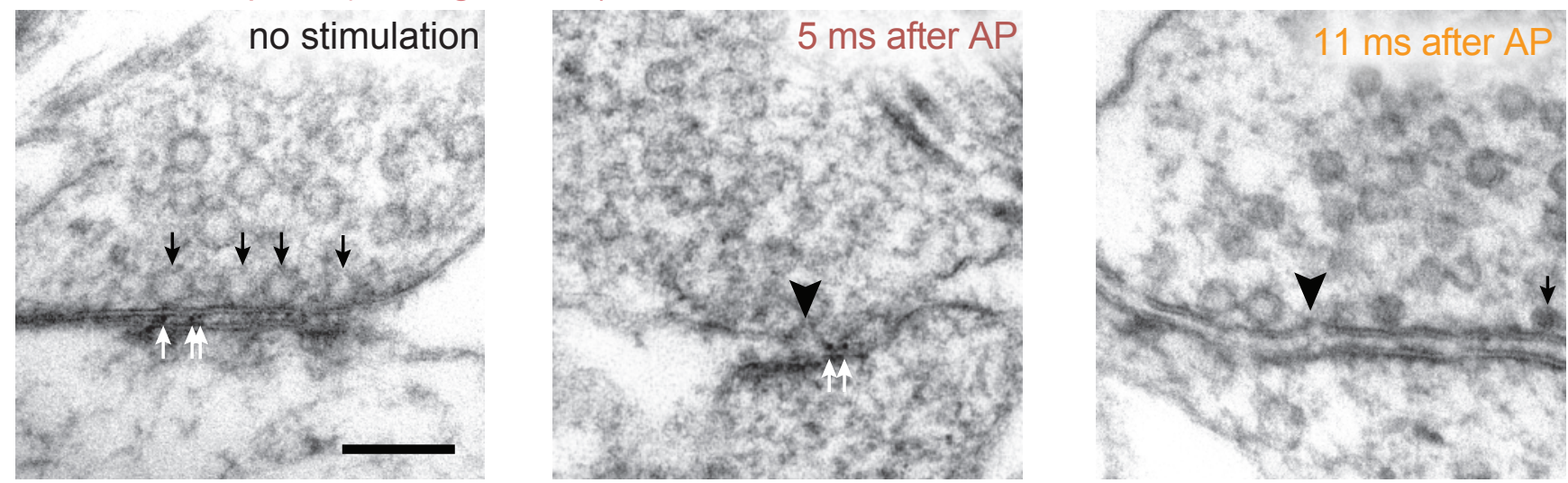

b NMDA receptors (His-tag::NR1)
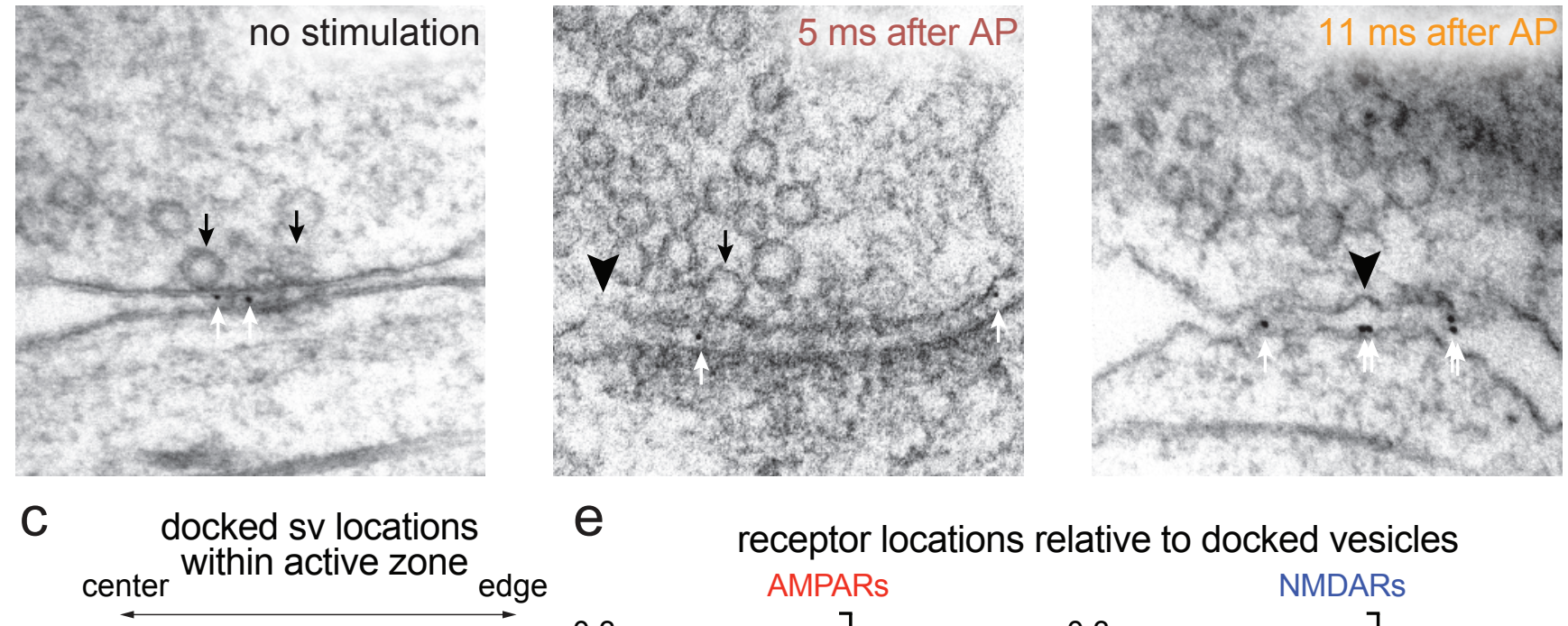

\section{receptor locations relative to docked vesicles}
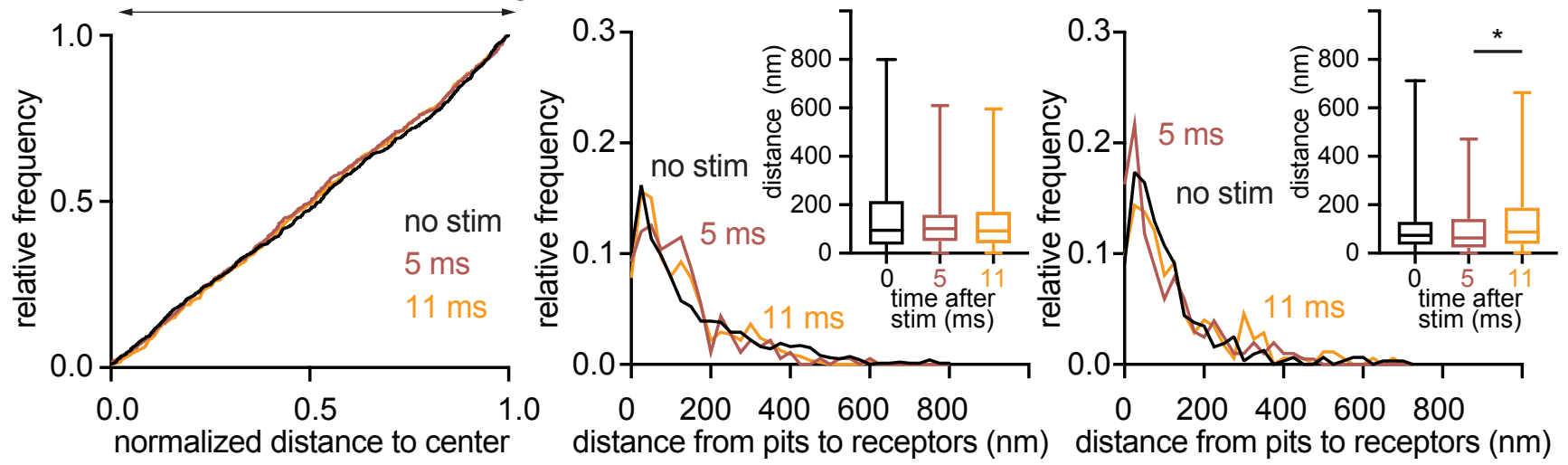

$\mathrm{d}_{\text {pit locations within active zone }}$
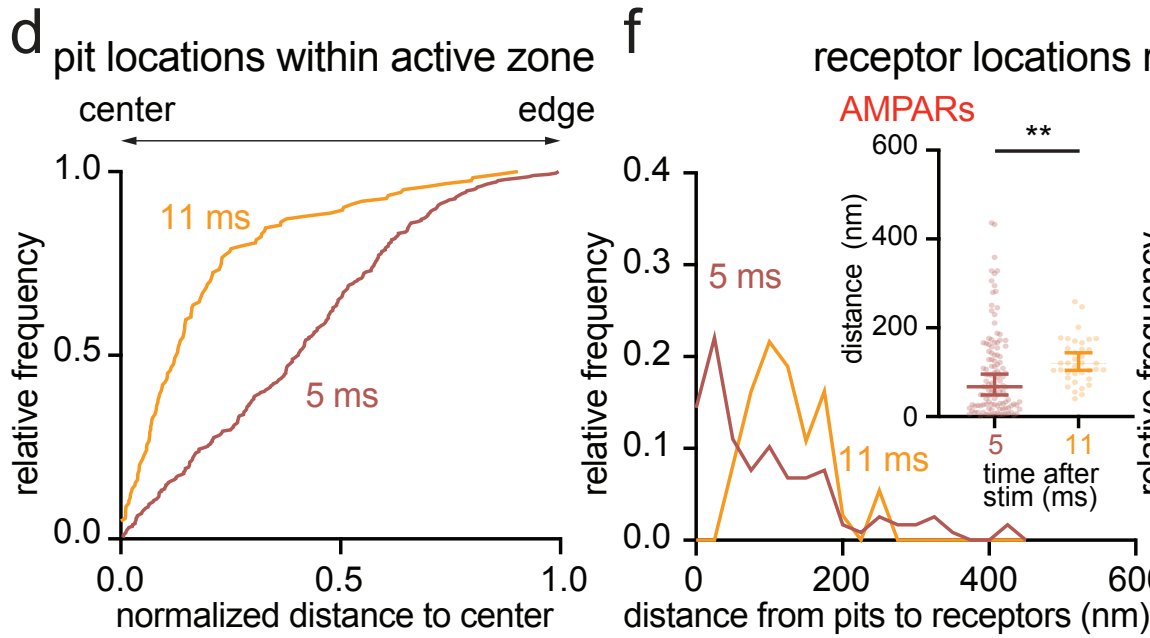

receptor locations relative to exocytic pits

distance from pits to receptors $(\mathrm{nm})$ distance from pits to receptors $(\mathrm{nm})$ 
Fig. 2. Synchronous and asynchronous release are aligned to AMPA receptors and NMDA receptors, respectively.

a-b, Example transmission electron micrographs of synapses after SMASH labelling and highpressure freezing at the indicated time points post stimulus (1-ms pulse), showing pits (black arrowheads), docked vesicles (black arrows) and gold particles (white arrows) at synapses of wild-type neurons expressing His-tag::GluA2 (a) and His-tag::NR1 (b). c-d, Cumulative relative frequency distribution of lateral distances between docked vesicles (c) or exocytic pits (d) and the active zone center at 5 or $11 \mathrm{~ms}$ after stimulation, as measured within 2-D profiles by transmission electron microscopy. Exocytic pits were biased towards the center both at $5 \mathrm{~ms}$ (median $=0.4, \mathrm{p}<0.001, \mathrm{n}=286$ pits, $\mathrm{N}=7$ samples $)$ and $11 \mathrm{~ms}($ median $=0.13, \mathrm{p}<0.001, \mathrm{n}=$ 124 pits, $N=7$ samples), but pits at $11 \mathrm{~ms}$ were more strongly biased towards the center ( $\mathrm{p}$ $<0.001$, Kolmogorov-Smirnov D test). Bias of vesicles and pits locations toward the center or edge of the active zone in $\mathbf{c}$ and $\mathbf{d}$ was tested by comparing each group to a theoretical median of 0.5 using one-sample two-tailed Wilcoxon signed-rank tests. e-f, Relative frequency distribution of lateral distances between docked vesicles (e) or exocytic pits (f) and gold particles within the cleft at $5 \mathrm{~ms}$ (red) and $11 \mathrm{~ms}$ (orange) after stimulation. Insets: the same data plotted as box and whiskers for docked vesicles (e) and scattered dots (f) to show the median distances. Error bars: median and $95 \%$ confidence interval for the scattered dot plots. Kolmogorov-Smirnov D tests were performed in each case, with additional post hoc Dunn's multiple comparisons tests for docked vesicle data. $* \mathrm{p}<0.05, * * \mathrm{p}<0.01,{ }^{* * *} \mathrm{p}<0.001$. See Supplementary Data Table 1 for full pairwise comparisons and summary statistics. 


\section{Fig. 3. Computer simulations predict better activation of NMDA receptors with}

\section{asynchronous release.}

a, Time course of simulated NMDA receptor activation, resulting from two release events at the indicated locations while varying the degree of depolarization of the postsynaptic membrane. The number averaged from 48 simulations are plotted. The vertical lines in the diagrams shown above the plot indicate when synchronous and asynchronous release occur. Synchronous release always occurs at time 0 . Double release means two release events happen simultaneously. Synch $=$ synchronous. Asynch $=$ asynchronous. $\mathbf{b}$, The area under curve calculated from each dataset in (a) and plotted as a bar graph. The locations of release are described in $\mathbf{a}$, and the degree of depolarization (depol) and the delay between synchronous and asynchronous release used in simulated are listed at the bottom. c, Same as a, but varying the interval between two release events. d, Same as b, but plotted from each dataset in (c). e, Same as in $\mathbf{a}$, but varying the order of the release. $\mathbf{f}$, Same as in $\mathbf{b}$, but plotted from each dataset in (e) and plotted as a bar graph. A$\mathrm{N}$ : synchronous release at AMPA receptors and asynchronous release at NMDA receptors. N-A: synchronous release at NMDA receptors and asynchronous release at AMPA receptors. N-N: both synchronous and asynchronous release at NMDA receptors. g, Schematic of proposed synaptic organization and events. Docked vesicles are found throughout the active zone. AMPA receptors are found towards the edge, while NMDA receptors are biased towards the center. Synchronous fusion begins within hundreds of microseconds near the AMPA receptor cluster. Released glutamate activates AMPA receptors, which in turn depolarizes the membrane and alleviates the $\mathrm{Mg}^{2+}$ block of NMDA receptors. Between 5 and $11 \mathrm{~ms}$, residual calcium triggers asynchronous fusion, preferentially toward the center of the active zone and across from the NMDA receptor cluster, favoring the NMDA receptors. Although shown here as taking place in 
the same active zone, the degree to which synchronous and asynchronous release may occur at the same active zone after a single action potential is unknown. This trans-synaptic organization allows the maximal depolarization of the postsynaptic membrane and efficient activation of NMDA receptors. 


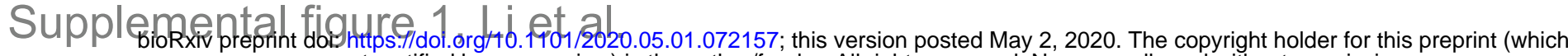
was not certified by peer review) is the author/funder. All rights reserved. No reuse allowed without permission.

a

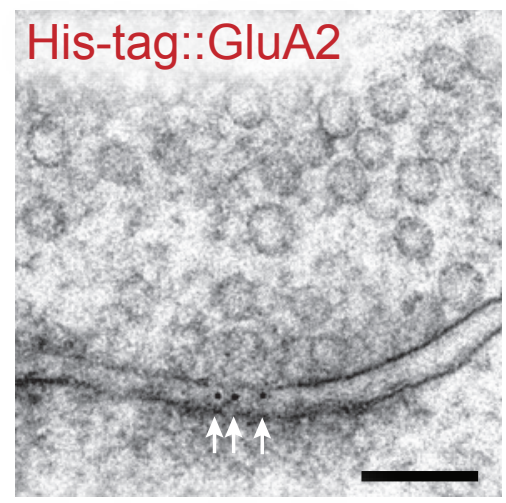

His-tag::GluA2

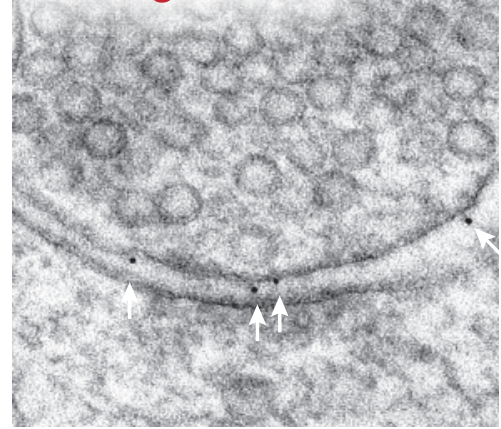

$\mathrm{C}$

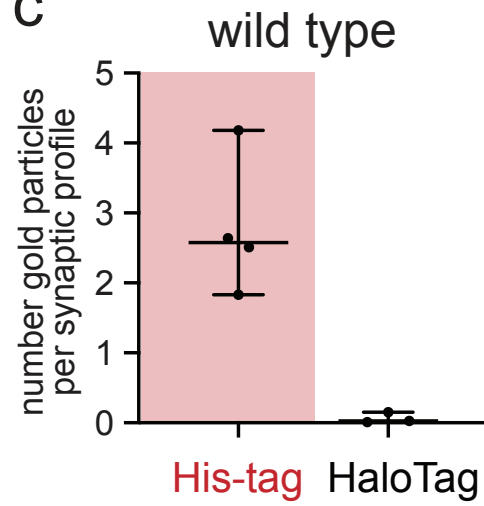

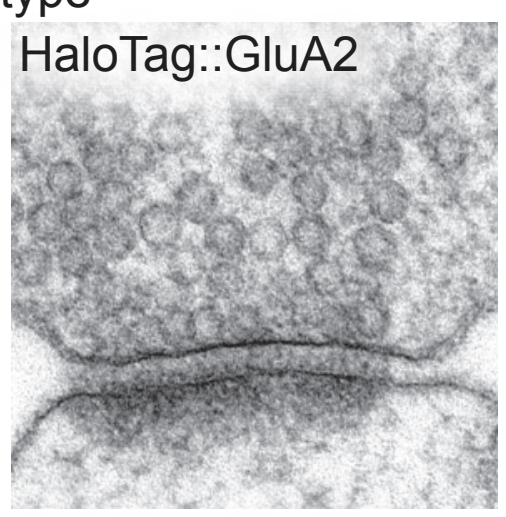
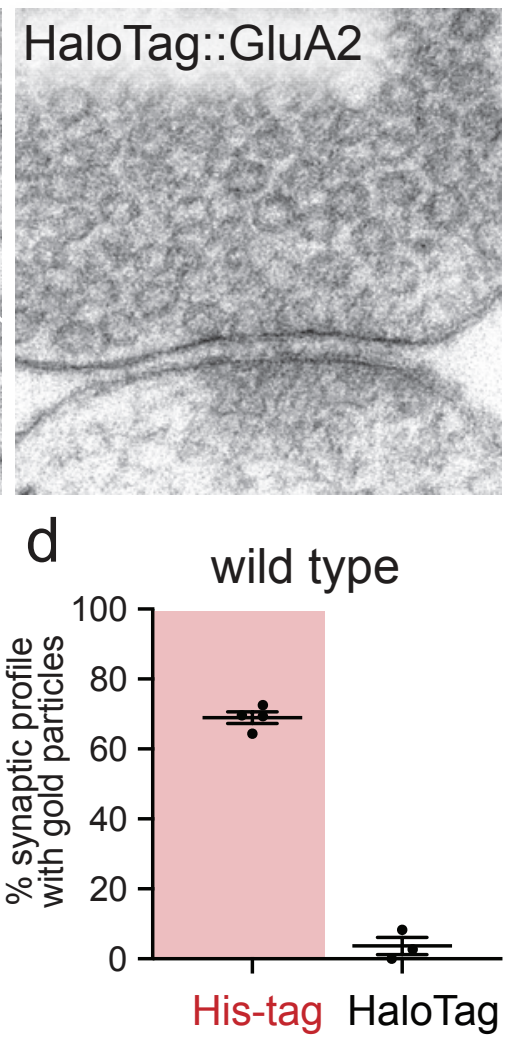

b
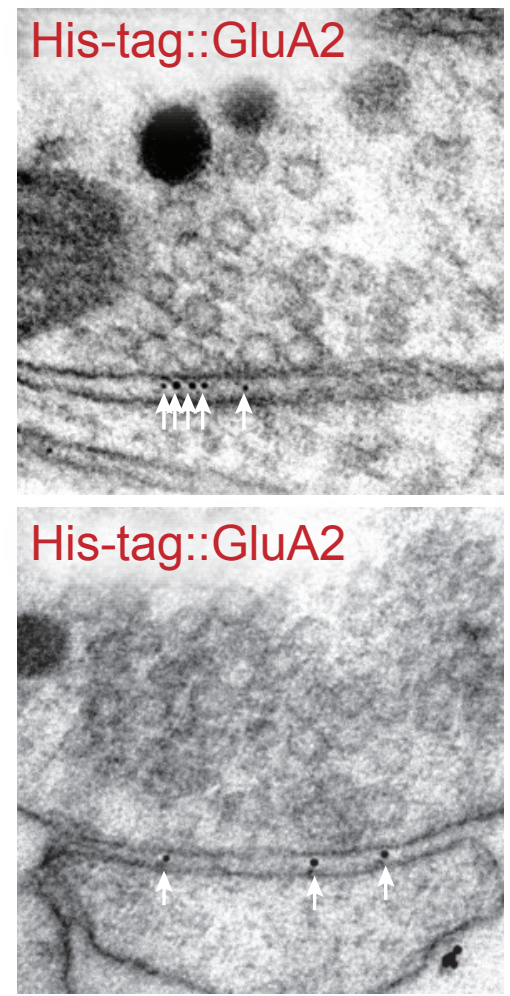

e

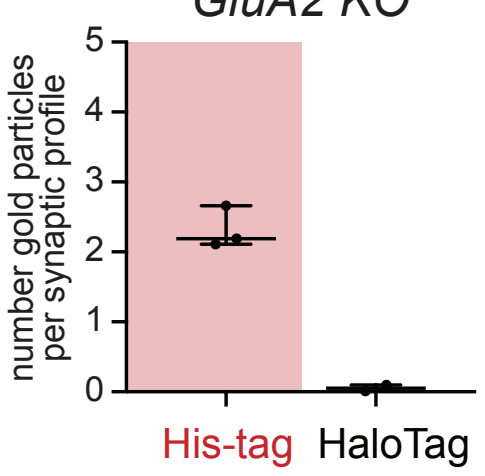

GluA2 KO

HaloTag::GluA2

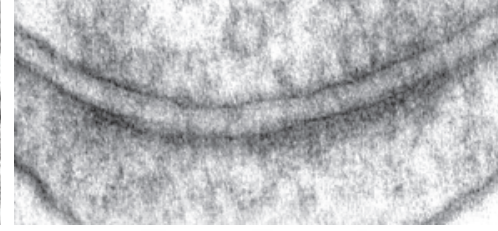

HaloTag::GluA2
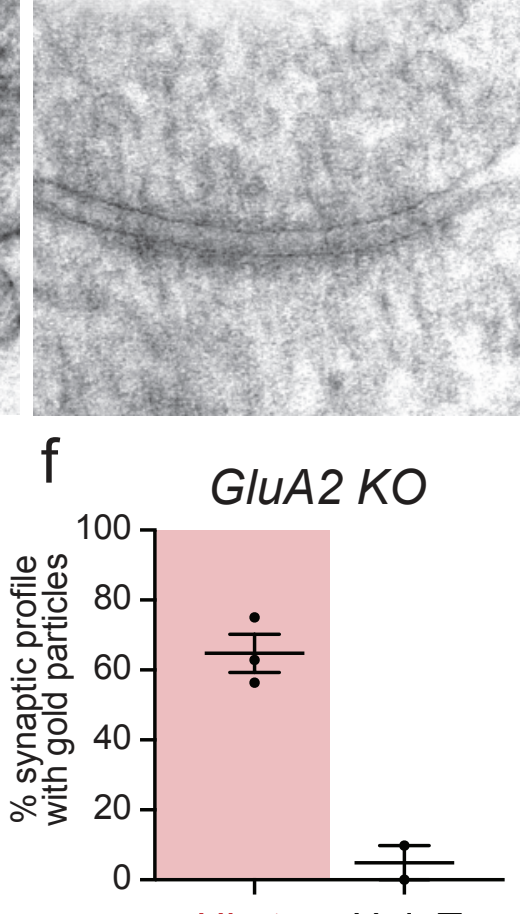

His-tag HaloTag 


\section{Supplementary Fig. 1. The receptor labelling approach and its validation}

a-b, Example transmission electron micrographs of synapses after SMASH labelling and highpressure freezing, showing gold particles in the synaptic cleft of wild-type (a) and GluA2 knockout (b, Gria2 $\left.2^{-/}\right)$neurons expressing His-tag::GluA2. Control samples with neurons expressing HaloTag::GluA2. Scale bar: $100 \mathrm{~nm}$. White arrows indicate gold particles. c, Number of gold particles per synaptic cleft in the wild-type neurons. Each dot: an average number from a single experiment, analyzing $\sim 100$ micrographs. Error bars: median and $95 \%$ confidence interval, $\mathrm{p}=0.01$, Welch's T-test. $\mathbf{d}$, Percentage of synaptic profiles containing gold particles in synaptic cleft in the wild-type neurons. Each dot: a percentage from a single experiment, analyzing $\sim 100$ micrographs. Error bars: mean and SEM, p<0.001, Welch's T-test. e, Same as in c, except showing the number from the GluA2 knockout (KO) neurons. Error bars: median and 95\% confidence interval, $\mathrm{p}<0.01$, Welch's T-test. f, Same as in $\mathbf{d}$, except showing the number from the GluA2 knockout (KO) neurons. Error bars: mean and SEM, p<0.01, Welch's T-test. 


\section{a}

\section{AMPA receptors}

\section{D reconstructions}
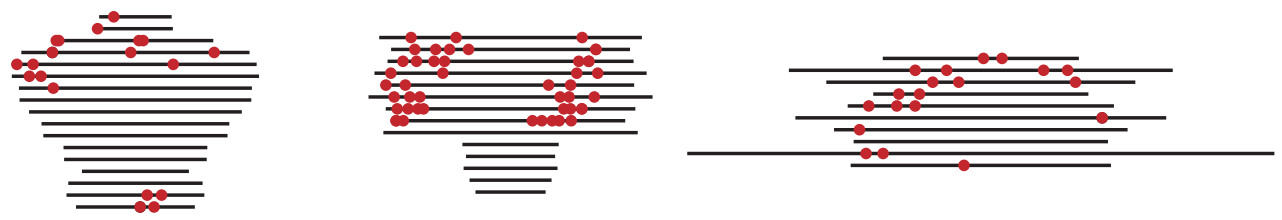

$\mathrm{b}$

$50 \mathrm{~nm}$
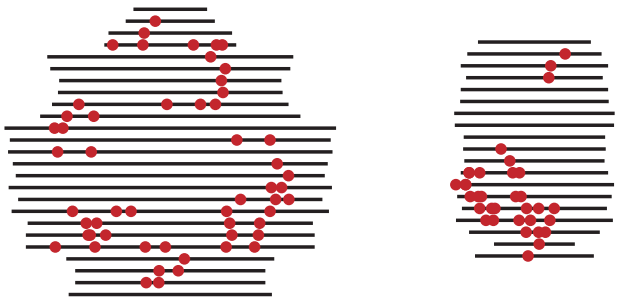

\section{K-means cluster analysis}
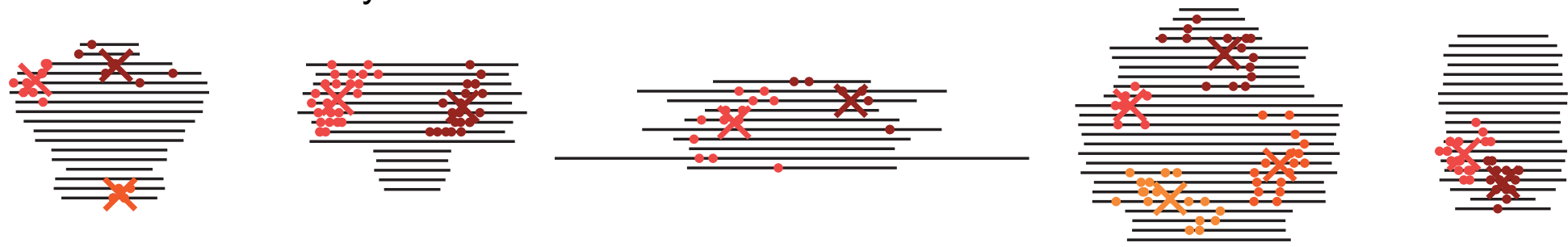

C

\section{NMDA receptors}

\section{$3 \mathrm{D}$ reconstructions}
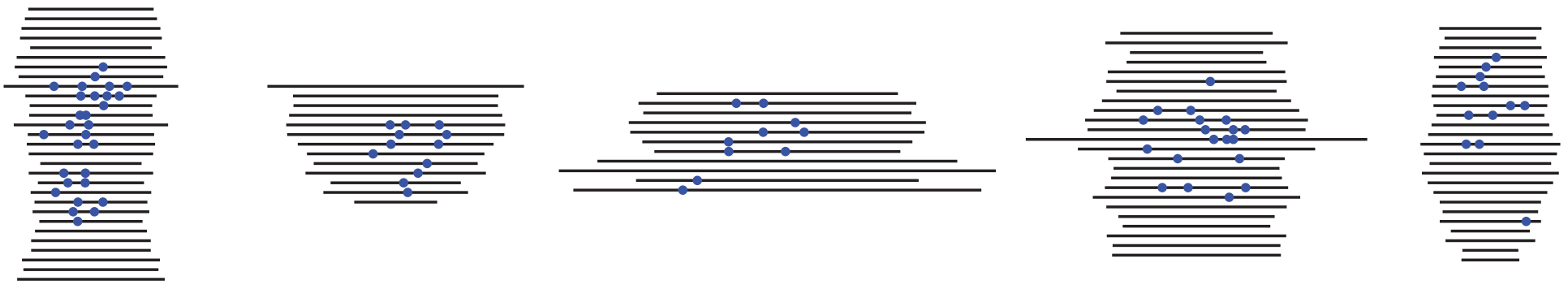

d

K-means cluster analysis
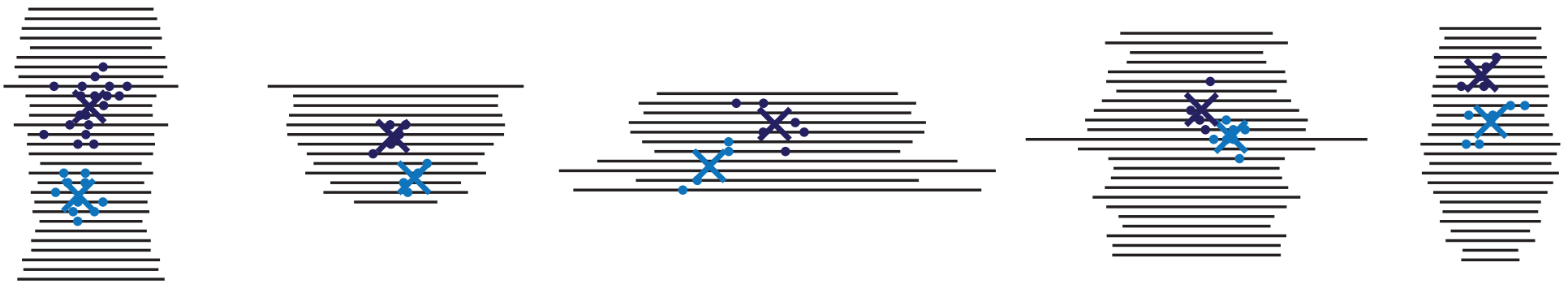


\section{Supplementary Fig. 2. Additional 3-D representations of AMPA receptors and NMDA receptors.}

a, Examples of synapses of neurons expressing His-tag::GluA2 from spin-mill serial block face scanning electron microscopy; each line indicates the extent of the cleft in a single $20 \mathrm{~nm}$-thick 2-D profile, each circle indicates the location of a gold particle. $\mathbf{b}$, Same as a, except showing the centers of clusters determined by k-means clustering. c, Examples of synapses of neurons expressing His-tag::NR1 from 3-D serial imaging; each line indicates the extent of the cleft in a single 20 nm-thick 2-D profile, each circle indicates the location of a gold particle. d, Same as c, except showing the centers of clusters determined by k-means clustering. 
bioRxiv preprint doi: https://doi.org/10.1101/2020.05.01.072157; this version posted May 2, 2020. The copyright holder for this preprint (which Supplementzyas nitygertified by peen rezieweis the author/funder. All rights reserved. No reuse allowed without permission.

a

AMPA receptors

5 ms after AP
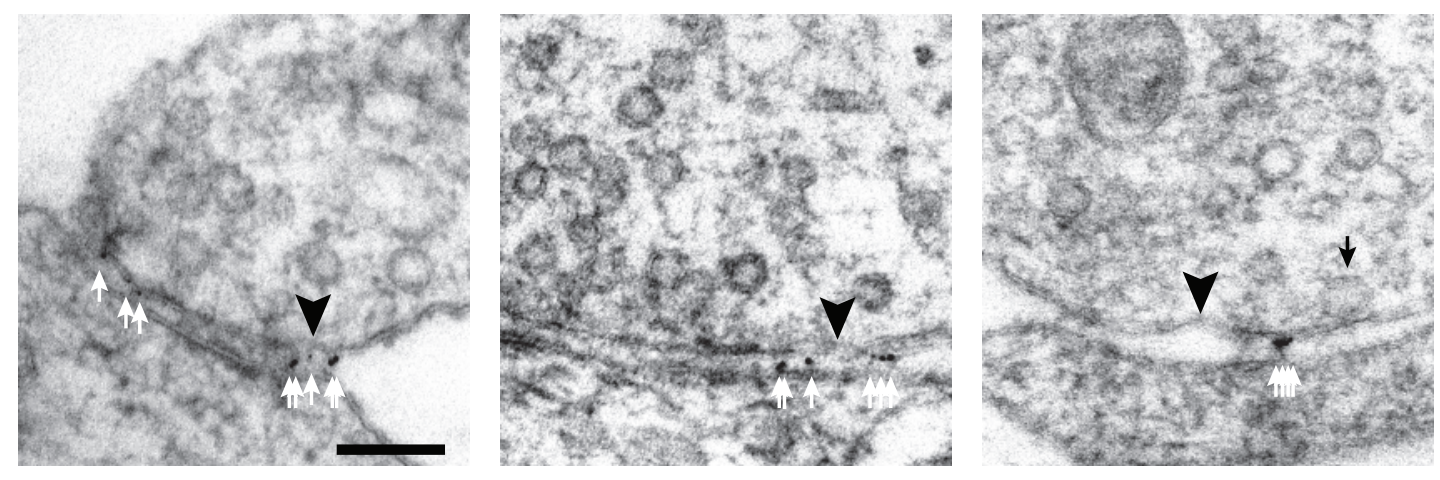

b

AMPA receptors

11 ms after AP
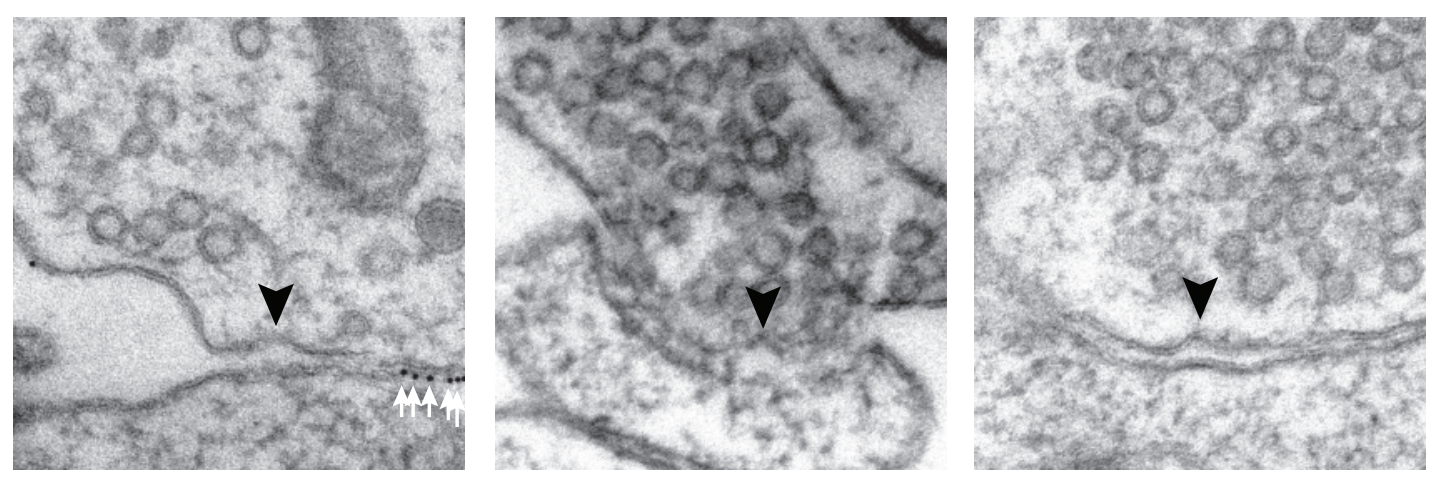

C

NMDA receptors

5 ms after AP
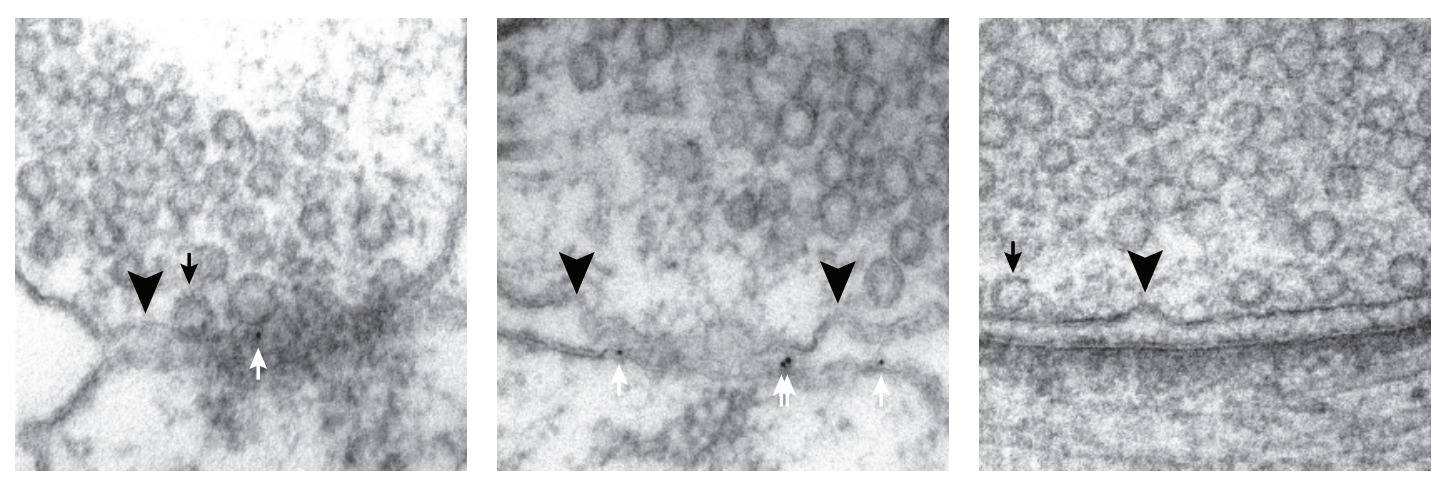

d
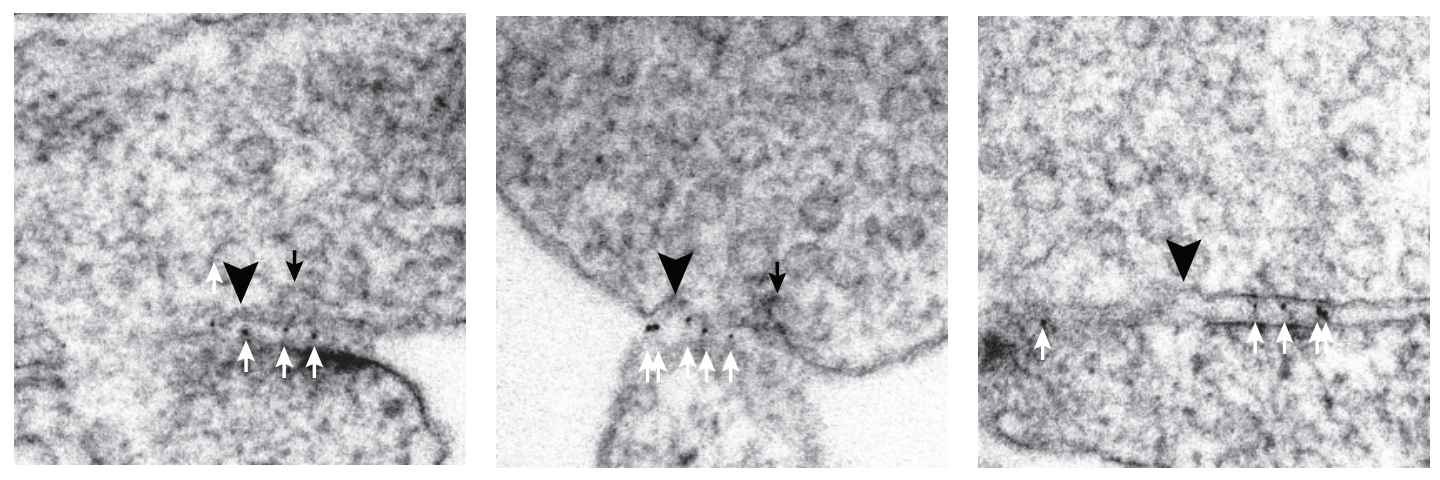

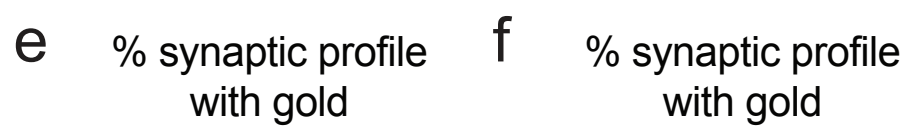

g docked sv \#

h

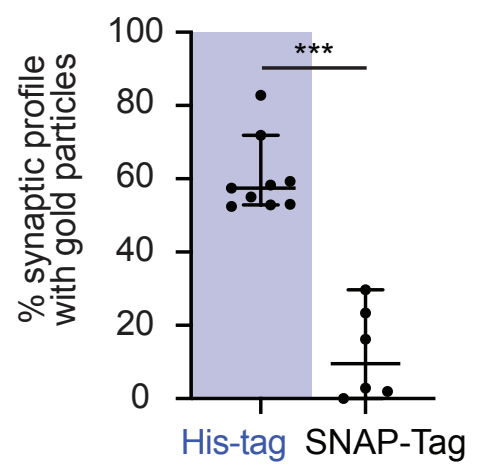

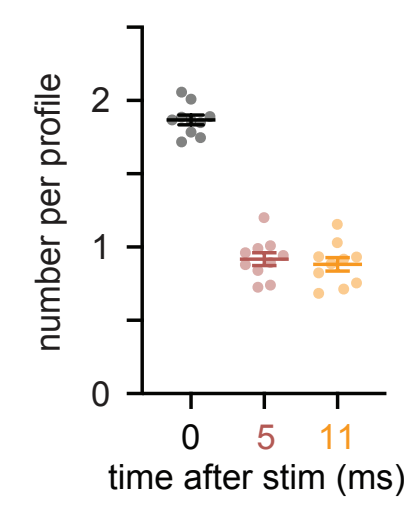

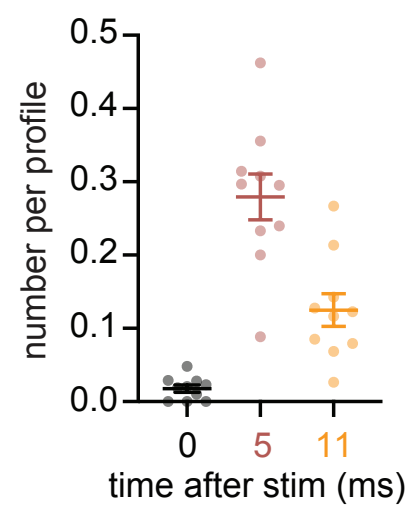

fusion pits \# 


\section{Supplementary Fig. 3. Synchronous and asynchronous release are aligned to AMPA receptors and NMDA receptors, respectively.}

a-d, Additional example transmission electron micrographs of synapses after SMASH labelling and high-pressure freezing at $5 \mathrm{~ms}(\mathbf{a}, \mathbf{c})$ and $11 \mathrm{~ms}(\mathbf{b}, \mathbf{d})$ post stimulus (1-ms pulse), showing pits (black arrowheads), docked vesicles (black arrows) and gold particles (white arrows) at synapses of wild-type neurons expressing His-tag::GluA2 (a-b) and His-tag::NR1 (c-d). e-f, Percentage of synaptic profiles containing gold particles in synaptic cleft in the wild-type neurons expressing His-tag::GluA2 or SNAP-Tag::GluA2 (e) and His-tag::NR1 or SNAPTag::NR1 (f). Each dot: a percentage from a single experiment, analyzing $\sim 100$ micrographs. Error bars: mean and SEM, p<0.001, Welch's T-test. g-h, Number of docked vesicles (g) and pits (h) in the active zone per synaptic profile from the neurons either unstimulated (black) or stimulated once (a 1-ms pulse) $5 \mathrm{~ms}$ (red) or $11 \mathrm{~ms}$ (orange) before freezing. Each dot: a mean

from a single experiment, analyzing $\sim 100$ micrographs. Error bars: mean and SEM, $<<0.001$ in all cases except for the numbers of docked vesicles between 5 and $11 \mathrm{~ms}(\mathrm{p}>0.6), 2$-way ANOVA with post hoc Turkey's multiple comparisons test. 
Supplemental figure 4. Li et al.

a

AMPA receptors

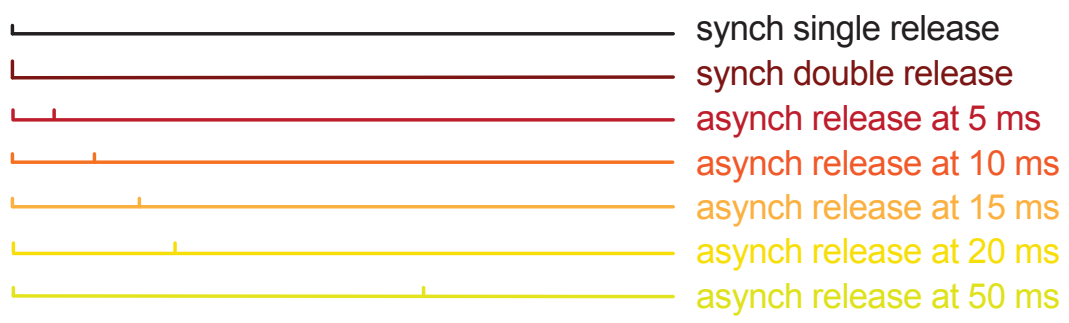

d NMDA receptors
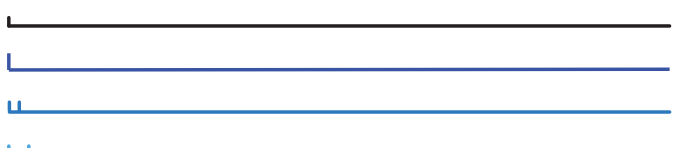

$\begin{array}{lll}1.1 & \text { asynch release at } 15 \mathrm{~ms} \\ & \text { asynch release at } 20 \mathrm{~ms}\end{array}$

asynch release at $50 \mathrm{~ms}$

e NMDA receptor response f NMDA receptor response
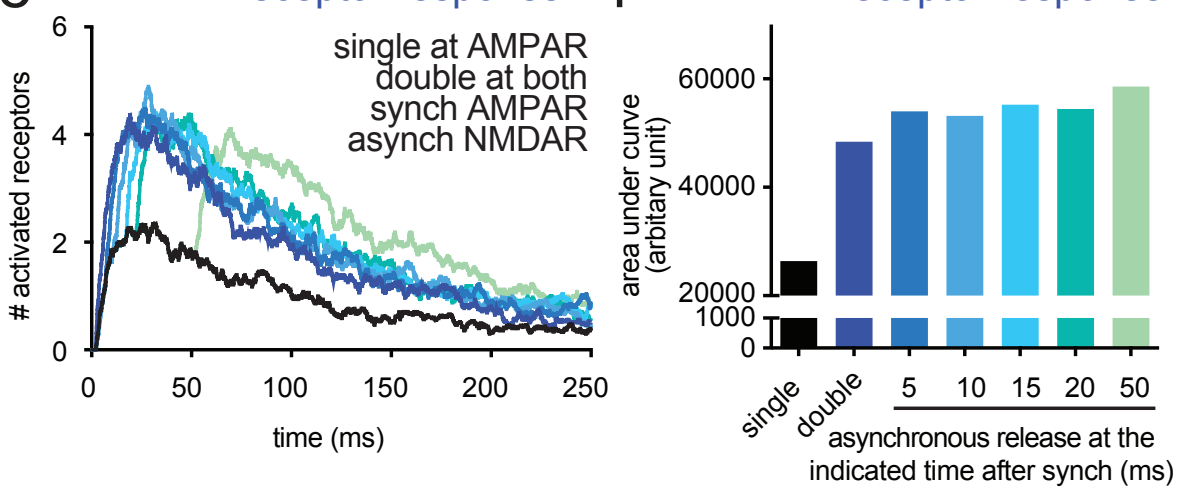

asynchronous release at the asynchronous release at the
indicated time after synch $(\mathrm{ms})$

g

simulations of membrane depolarization after glutamate release from a single vesicle

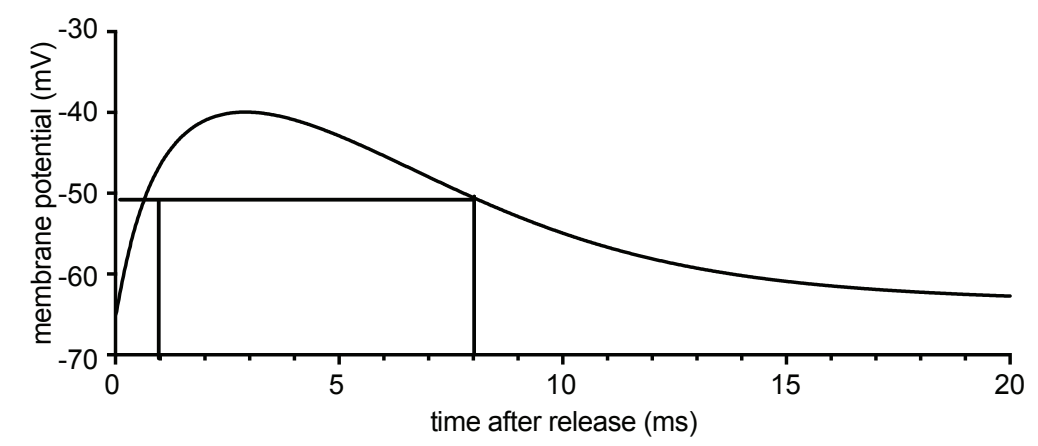

h NMDA receptor response

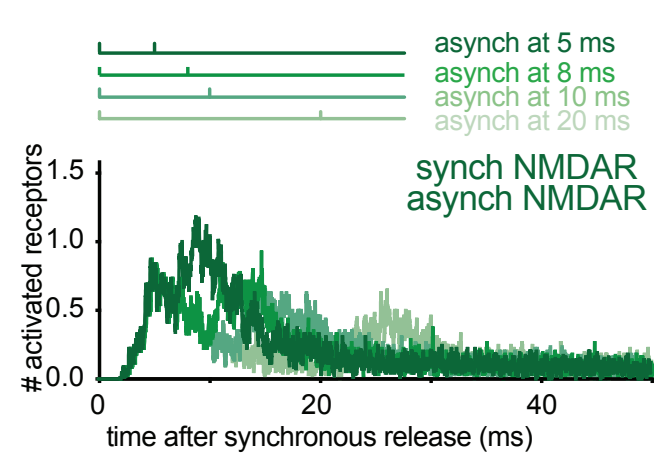

i

NMDA receptor response synch AMPAR synch AMPAR
synch NMDAR asynch NMDAR NMDAR
asynch NMDAR

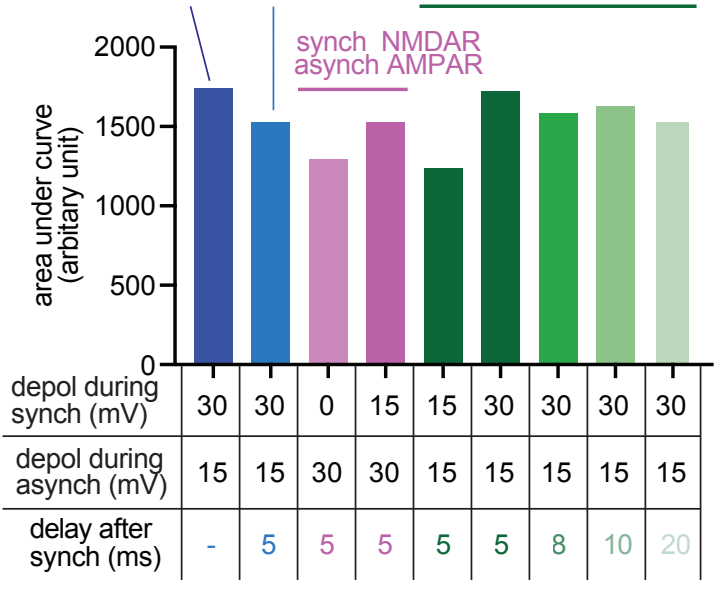




\section{Supplementary Fig. 4. Computer simulations predict better activation of NMDA receptors with asynchronous release.}

a-b, Time course of simulated AMPA receptor activation in the absence of $\mathrm{Mg}^{2+}(\mathbf{b})$, resulting from release events at the timing and locations as indicated in (a) and (b), respectively. The vertical lines in (a) indicate when synchronous and asynchronous release was applied. The number averaged from 48 simulations are plotted in (b). The synchronous release was applied at time 0 . Double release means two release events were applied simultaneously. Synch $=$ synchronous. Asynch = asynchronous. c, the area under curve calculated from each dataset in (b) and plotted as a bar graph. d-e, same as in $\mathbf{a}-\mathbf{b}$, but for NMDA receptors. f, same as in $\mathbf{c}$ but for NMDA receptors. $\mathbf{g}$, Time course of simulated membrane depolarization due to the activation of AMPA receptors. The depolarization peaks between 3 to $5 \mathrm{~ms}$ after the glutamate release, declines to $2 / 3$ of the maximum after $8 \mathrm{~ms}$, and is almost back to the baseline by $10 \mathrm{~ms}$. $\mathbf{h}$, Time course of simulated NMDA receptor activation in the presence of $\mathrm{Mg}^{2+}$, resulting from both synchronous and asynchronous release occurring near the NMDA receptors. The membrane depolarization due to synchronous release was simulated at $30 \mathrm{mV}$. i, The area under curve calculated from each dataset in (h) and plotted as a bar graph. The locations of synchronous and asynchronous release are as indicated. The degree of depolarization (depol) and the delay between synchronous and asynchronous release used in simulated are listed at the bottom. 